\title{
WestVirginiaUniversity
}

THE RESEARCH REPOSITORY @ WVU

Graduate Theses, Dissertations, and Problem Reports

2014

\section{Acute Sleep Organization Following Unanesthetized Circumcision of Neonates}

\author{
Margeaux M. Gray \\ West Virginia University
}

Follow this and additional works at: https://researchrepository.wvu.edu/etd

\section{Recommended Citation}

Gray, Margeaux M., "Acute Sleep Organization Following Unanesthetized Circumcision of Neonates" (2014). Graduate Theses, Dissertations, and Problem Reports. 205.

https://researchrepository.wvu.edu/etd/205

This Thesis is protected by copyright and/or related rights. It has been brought to you by the The Research Repository @ WVU with permission from the rights-holder(s). You are free to use this Thesis in any way that is permitted by the copyright and related rights legislation that applies to your use. For other uses you must obtain permission from the rights-holder(s) directly, unless additional rights are indicated by a Creative Commons license in the record and/ or on the work itself. This Thesis has been accepted for inclusion in WVU Graduate Theses, Dissertations, and Problem Reports collection by an authorized administrator of The Research Repository @ WVU. For more information, please contact researchrepository@mail.wvu.edu. 


\title{
Acute Sleep Organization Following Unanesthetized Circumcision of Neonates
}

\author{
Margeaux M. Gray, B.S. \\ Thesis submitted \\ to the Eberly College of Arts and Sciences \\ at West Virginia University \\ in partial fulfillment of the requirements for the degree of \\ Master of Science in \\ Psychology (Behavioral Neuroscience)
}
Hawley Montgomery-Downs, Ph.D., Chair
Steven Kinsey, Ph.D.
Daniel W. McNeil, Ph.D.
Department of Psychology

Morgantown, West Virginia

2013

Keywords: Neonate, Sleep, Circumcision, Quiet Sleep, Transitional Sleep, Respiratory Rate, Motility Monitoring

Copyright 2013 Margeaux M. Gray 


\title{
ABSTRACT \\ Acute Sleep Organization Following Unanesthetized Circumcision of Neonates
}

\begin{abstract}
Margeaux M. Gray
Sleep changes in response to environmental pressures and the needs of individuals. The adaptability of sleep is noticeable following largely stressful or adverse events, and is understood to facilitate recovery. Rapid changes in sleep occur in-utero, during the days following birth, and across the first few postnatal years. These rapid changes are considered adaptive and indicative of normal and healthy development. Elective surgical intervention, such as circumcision, may influence the organization and quality of sleep during this developmentally sensitive period. Neonates undergoing circumcision, a prevalent procedure in the U.S. (American Academy of Pediatrics Circumcision Policy Statement [AAP], 1999, 2005, 2012), also are at risk for operative stress or postoperative pain. Changes in the sleep organization of neonates have been observed following circumcision, but are not consistent. In these analyses, I specifically evaluated changes in infant sleep cycles surrounding circumcision from pre-existing data. These data present a unique opportunity to characterize sleep in relation to circumcision because they were collected prior to routine use of anesthetic or analgesic. Infants were compared on sleep state organization, determined using Motility Monitoring recordings of infant movement and respiration. Quiet sleep of circumcised infants decreased and transitional sleep marginally increased from before circumcision to after circumcision. Respiratory rate during quiet sleep increased among both circumcision and control groups. The lapse from active sleep to quiet sleep decreased for uncircumcised males and did not change for the circumcised group. Male neonates appear to demonstrate Quiet and Transitional sleep trends from pre- to post-circumcision, but ultimately neither of their changes differed in comparison to a group of combined females and uncircumcised males. It is plausible that test outcomes did not reach statistical significance because of differences in the distribution of infant sex across groups, or because of small sample size. Although no causal relation between circumcision and sleep measures can be inferred from these data, the changes in sleep organization that were demonstrated by circumcised males in this study should be investigated among infants receiving procedural anesthesia, but who do not typically receive analgesic intervention to mediate postoperative pain's effect on sleep during recovery after circumcision.
\end{abstract}




\section{Table of Contents}

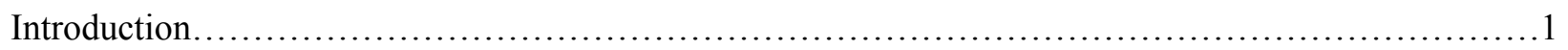

Infant Sleep Architecture and Behavioral Assessment.....................................2

Circumcision..................................................................4

Changes in Infant Sleep Organization Following Physical Trauma or Stress..................6

Infant Nociceptive Responses and Sleep............................................

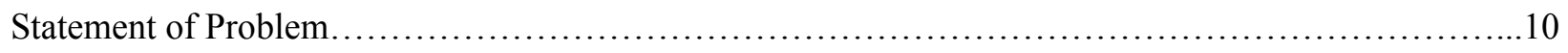

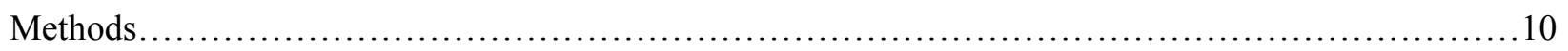

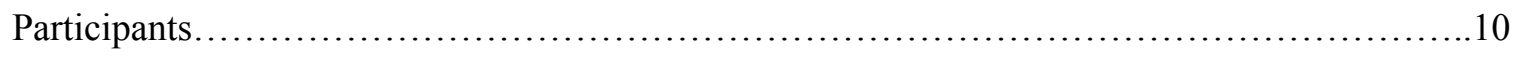

Circumcised Infants.................................................... 11

Control Infants.........................................................11

Procedure....................................................................... 12

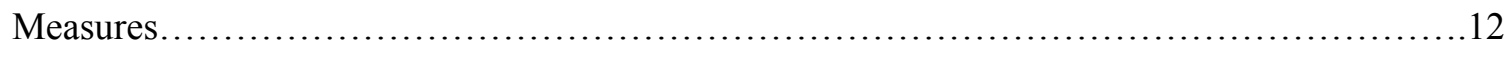

Data Analysis...............................................................

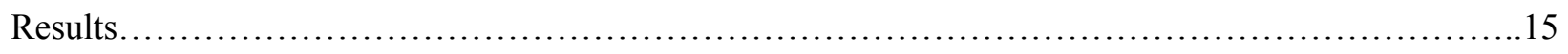

Active Sleep, Quiet Sleep, and Transitional Sleep....................................15

Latency to Quiet Sleep........................................................... 19

Transition Index .............................................................21

Quiet Sleep Respiratory Rate................................................... 24

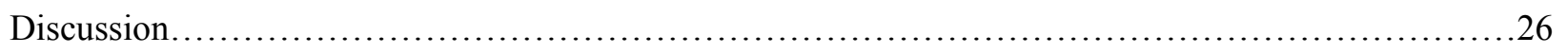

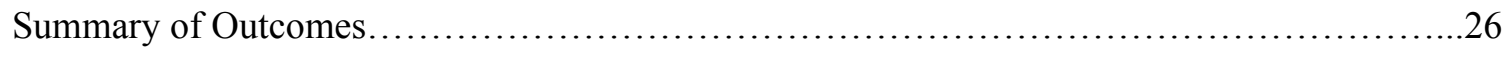

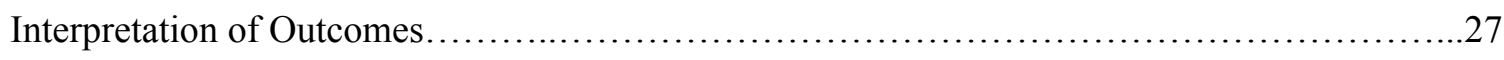

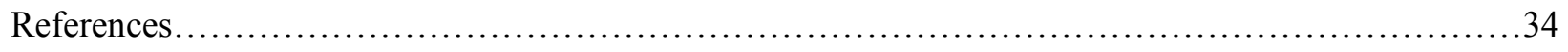

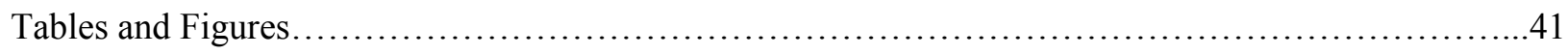




\section{Acute Sleep Disruption Following Unanesthetized Circumcision of Neonates}

Sleep is a consistently recurring set of behavioral and physiological states characterized by reduced arousal, yet is distinct from unconscious states in its easy reversibility. It is dynamic despite its quiescent appearance in adults, varying both within a night and from night-to-night based on our body's needs, and reflecting developmental changes in quality and quantity across the lifespan. In consideration of sleep's changes across age, it would be appropriate to suspect that the elements of sleep undergoing change correspond to biological needs during particular phases of development. Some of the most rapid and dramatic changes in sleep behavior occur during the first year of life. It may be that disturbing sleep during a developmentally sensitive period has adverse effects on growth or health maintenance. Rodents with total sleep deprivation experience unmanageable weight loss and eventually death from unknown cause presenting as sepsis (Everson, Bergman, \& Retschaffen, 1989). Even with limited sleep restriction human immune function is compromised (Oztürk et al., 1999). While reverting back to an awake state with ease has adaptive advantage in the event of environmental insult, a balance must be mediated endogenously to avoid excessive arousal from sleep or otherwise risk susceptibility to infection. Sleep quantity corresponds to early physical growth (Lampl \& Johnson, 2011). Sleep also is heavily targeted in its role facilitating learning and memory (Tarullo, Balsam, \& Fifer, 2011). In fact, infants demonstrate learning during sleep itself (Fifer et al., 2010). Indicators of poor infant sleep quality (like snoring) precipitate poorer developmental outcomes (Piteo et al., 2011). Variations in sleep-related respiratory rate at 6 months of age are related to developmental Bayley scores at 6 months and at 1year (MontgomeryDowns \& Thoman, 1998). Infants may not recover completely from sleep interruption that occurs during a developmentally sensitive window, and so causes of even acute sleep disturbance deserve special attention in their case.

While sleep interruption as a result of respiratory disturbance (as upper airway occlusion) or congenital syndromes (as comorbidities) has been investigated in the pediatric population, disturbances related to pain have been largely overlooked. This may in part be due to difficulty quantifying pain in general, especially the pain of very young children even while they are awake. Limitations of our ability 
to detect their pain don't preclude considering the impact of procedures suspected to cause infant pain, or their influence on infant sleep, however. Invasive procedures deserve special consideration in relation to neonatal sleep because pain is known to adversely impact adult sleep (Dauvilliers \& Touchon, 2001). Specifically, circumcision is an elective practice in the U.S. and in many other nations that has received special attention within the last twenty years. It is historically the most common pediatric surgical intervention, and dates back to Egyptian tradition (World Health Organization [WHO], 2010). As of 2005, the AAP (for legend of acronyms, see Figure 1) reported that about 1.2 million circumcisions occur in the U.S. annually, affecting upwards of $60 \%$ of male infants (although the proportionate statistics vary ethnically and regionally; AAP, 1999, 2005). Recent concerns addressed the risks, benefits, and the appropriate medical interventions to mitigate pain caused during the circumcision procedure itself. Clarification of its influence on neonatal sleep is the aim of the present study.

Nonintrusive macro-observation techniques were used in a clinical setting to obtain data from circumcised and uncircumcised neonates during previous investigations, and these data were reevaluated objectively from both behavioral and physiological perspectives to determine circumcision's influence on infant sleep quality. However, to recognize abnormalities in the infants' sleep, and to distinguish those events potentially related to circumcision or pain, one must first consider the characteristics of typical infant sleep and nociception. These topics are briefly elaborated upon below, in relation to circumcision/physical trauma and in relation to the Motility Monitoring System (MMS) methodology utilized in infant behavior quantification.

\section{Infant Sleep Architecture and Behavioral Assessment}

Neonatal sleep differs in organization from the sleep of adults, in that it follows an ultradian, rather than circadian, rhythm. Their polyphasic sleep episodes are 45-60mins in duration (Sheldon, 2005) and are comprised of cyclically alternating sleep states, totaling upwards of $12 \mathrm{hrs}$ of sleep in a $24 \mathrm{hr}$ period (Montgomery-Downs \& Gozal, 2006). Gradually, the behaviors of caregivers and light exposure influence endogenous regulation of infant sleep, bringing it closer to a circadian rhythm by 3-4 months of 
age (Herman, 2005; Anders, 2010). Sleep episode distribution only reveals superficial differences between infant and adult sleep; however, sleep stage regulation within episodes shows considerable internal variation from that of adults.

Infant sleep cycles initiate with rapid transition from a drowse state to Active Sleep (AS) until the infant is about 3 months old, when the Non-Rapid Eye Movement (NREM) sleep onset typical of adults is observed (Korner, 1968). Proportionately, close to half of newborn sleep is spent in the Active state and gradually decreases throughout the first year of life to adult-like levels of Active Sleep's equivalent, Rapid Eye Movement (REM) sleep. Conversely, Quiet Sleep (QS) occupies the other infant sleep time and is comparable to NREM sleep in adults both physiologically, with stable respiration (Korner, 1968; Sheldon, 2005; Sorensen, Carskadon, \& Ursin, 2007) and little movement aside from sudden, dramatic startle responses (Korner, 1968, 1969; Sitka, 1990). Occasionally, infant sleep state can be difficult to identify as specifically Active or Quiet, even with EEG and behavioral data available (especially during transitions between sleep states). So, Indeterminate Sleep (IS) is used to classify sleep demonstrating “mixed-state characteristics" (Korner, 1968; Sheldon, 2005, p. 52).

Although the QS of infants shares many overt characteristics with adult NREM sleep, AS dramatically differs from its analog, REM, in adults. The adult brainstem (the supraolivary nucleus of the medulla in rodents and cats, specifically; Vetrivelan, Fuller, Tong, \& Lu, 2009) attenuates muscle tone during REM sleep to prevent sleep behaviors. Infants' medullary components that regulate atonia are still underdeveloped compared to adults, though. This results in maintained tonus and very expressive AS, revealing AS-related behaviors above and beyond characteristic rapid eye movements and variable respiration (Korner, 1968; Sheldon, 2005) that can be used to help identify their behavioral state.

Because infant sleep states differ so dramatically, macroscopic observational methods of sleep quantification are not only feasible and reliable, they're preferable to more intrusive methods that may interfere with sleep (like EEG instrumentation). Behavioral observation to classify infant sleep state was first introduced as a reliable measurement in 1976, when Anders and Sostek reported strong correlations and high inter-rater reliability with EEG/Polygraphic techniques. Methods sensitive enough to record the 
increased movement from infant behavioral expression and their physiological changes in respiratory pattern now provide a valid and reliable resource for characterizing infant sleep without requisite human observation (Thoman \& Tynan, 1979). One such resource, the Motility Monitoring System (MMS), successfully discerns infant AS, QS, and TS (transitional, or indeterminate sleep) by using a pressuresensitive pad in the infant's crib or basinet. Recorded activity is converted from analog to digital in real time and is verified by a human scorer for accuracy against respiratory data, subverting observer reliability risk as a highly objective measure. As such, the MMS achieves a continuous characterization of infant sleep architecture without interfering with infant sleep quality (1979).

Another salient feature of AS in infant males, and of REM sleep in adults, is nocturnal penile tumescence (NPT). Although typical in AS/REM for both age groups, infant NPT occurs more often both overall and during the NREM-comparable state (QS) than it does in adult males (Karacan, Marans, Barnet, \& Lodge, 1968; Korner, 1968; Korner, 1969) - an interesting phenomenon on its own, but imperative to recognize when considering the effects it might have in relation to genital surgery. Diffuse

NPT occurring primarily during sleep lends a mechanism by which circumcision might affect infant sleep quality. While the MMS does not have NPT identification among its capabilities, it can be used to distinguish sleep states during which NPT is more pervasive and also changes in respiration that are associated with arousal from sleep (McNamara, Wulbrand, \& Thach, 1998).

\section{Circumcision}

For most of the latter part of the $20^{\text {th }}$ century, circumcision in the U.S. was performed while infants were completely unanesthetized, influenced by Flechsig's proposition in 1872 that infants do not feel pain because of incomplete myelination of nerve endings (Lee, 2002). Clinical concerns for infants' ability to feel pain emerged in the late 1980s through 1990s (Stang et al., 1978; Stang, Gunnar, Snellman, Condon, \& Kestenbaum, 1988; Porter, Wolf, Gold, Lotsoff, \& Miller, 1997; Porter, Wolf, \& Miller, 1999), and the medical profession rapidly adopted new circumcision practices as policy statements, in response (AAP, 1999). Cultural and international precedent for use of local anesthesia still varies, 
however (AAP, 1999, 2005), leaving considerable room for the impact of the proposed study on global circumcision practice. Conventional best practices for clinical circumcision in the U.S. now include local anesthetic (Dorsal Penile Nerve Block, Soh, Ng, \& Lim, 2003) and Eutectic Mixture of Local Anesthetics (EMLA) Cream (Benini, Johnston, Faucher, \& Aranda, 1993) among other interventions (Ring Block; AAP, 1999; Lander, Brady-Fryer, Metcalfe, Nazarali, \& Muttitt, 1997), and oral sucrose as an analgesic (Ramenghi, Wood, Griffith, \& Levene, 1996). EMLA’s successful effects are contingent upon appropriate administration, which allows it adequate penetration time up to a maximum of $10 \mathrm{~mm}$ for a few minutes' duration (Mathew \& Mathew, 2003) - qualities that render it inadequate if used alone (Taddio, 2001). Still, so long as interventions are used in practice (use remained inconsistent through 2005, Stang et al., 1988; Anand et al., 2005; Mathew \& Mathew, 2003), there is considerable comparative improvement in pain versus unanesthetized circumcision.

The most recent American Academy of Pediatrics Policy Statement advocates neonatal circumcision as a provision of general health benefit, so long as clinical best practices for anesthesia are adhered to, and therefore sets a precedent for circumcision advocacy in the clinical setting (2012). The guidelines highlight the potential long-term health benefits associated with circumcision, with a focus on sexually transmitted disease prevalence or incidence. There is some research that raises concern for adverse long-term effects of circumcision, however. Circumcised males show behavioral evidence of hyperaglesia when immunized several months later (Taddio, Katz, Ilersich, \& Koren, 1997), but hyperalgesia at immunization was not evident when effective analgesics were used during early surgical procedures (Peters, et al. 2003). Circumcised infants also have lower vagal tone during invasive procedures than uncircumcised infants, even when their circumcision involved lidocaine anesthesia (Gunnar et al. 1995). Circumcised infants' vagal tone was later associated with a less well-developed response to stress at 6 months (1995).

Postoperative circumcision care hasn't been awarded the same level of critique as procedural care. At most, infants receive analgesics like acetaminophen during the immediate postoperative period (Taddio, 2001; WHO, 2010). Infants are discharged from the hospital within a few days after 
circumcision, rendering them unlikely to receive consistent analgesic intervention across the duration of the healing process (5-7 days of superficial healing and 14 days until complete healing, WHO, 2010; Geyer, et al., 2002). It is unknown whether infants might experience pain as a result of wound aggravation during episodes of NPT in sleep, which could increase analgesic demand beyond what is necessary to relieve residual waking nociception. Pain-related sleep disturbance during the circumcision healing process has suffered the same inattention as postoperative analgesia in the research community. If extended postoperative intervention occurs, the regularity of its application hasn't been evaluated, and it's unlikely that it extends much beyond the hospital stay (Geyer, et al., 2002). Acknowledging this shortcoming reveals the additional, translational application the present research has for contemporary practice: even males circumcised today who are provided with procedural anesthetics may experience postoperative pain or sleep disturbance after being discharged.

Circumcised infants in the present sample were not provided pre-, intra-, or post-operational anesthesia or analgesia. Their experience of the pain associated with circumcision was unmitigated and any effects of the procedure or its sequelae on their sleep were unadulterated by medication, making them ideal examples of surgical pain's influence on sleep quality.

\section{Changes in Infant Sleep Organization Following Physical Trauma or Stress}

Infant (as well as other neonatal mammals), QS increases on the day of their birth (Carroll, Denenberg \& Thoman, 1999) could reflect a response to the intensity of the birthing process and the remarkable amount of adaptive demand on the body following birth (Lagercrantz \& Slotkin, 1986). Similarly, circumcision is physically traumatic, particularly in the absence of local anesthetic and activation of the Hypothalamic-Pituitary-Adrenal (HPA) response (Anand, et al., 1985). It's reasonable to hypothesize that similar changes in sleep architecture would be instigated by the two.

Infants circumcised without anesthesia have demonstrated QS increases of over $10 \%$ during the 48hrs following the procedure, while uncircumcised infants experience slight increases in AS proportionate to total sleep time (TST, about 4\%; Emde, Harmon, Metcalf, Koenig, \& Wagonfeld, 1971). 
Little change in the proportion of AS by circumcised, or QS by uncircumcised, infants was observed, but the circumcised infants' TST increased. Greater increases in QS than in AS when TST increases is counter-intuitive based on normal neonatal sleep architecture, suggesting that either the experience of circumcision is influencing sleep in a unique way, or that it is interfering with normal recovery of sleep following birth (by diminishing back to an expected 50\% QS; Dixon, Snyder, Holve, \& Bromberger, 1984). Emde et al. (1971) suspected that there was congruency between their findings and a proposed conservation-withdrawal (see 'depression-withdrawal,' and ‘conservation-withdrawal,' Engel, 1962; Engel \& Schmale, 1972; Kaufman \& Rosenblum, 1967) state, supposing that QS is serving as a protective mechanism to buffer the infant's sensitivity to pain or trauma. A reduced sleep-onset latency following circumcision (Emde, et al. 1971) also suggested sleep 'pressure,' or that infants' bodies had some increase in their homeostatic need for sleep that was greater than the uncircumcised infants' needs. Endogenous regulation of infant sleep appears to be mediated by physical trauma, pain, or stress, such that either the circumcision procedure itself or residual pain while healing might specifically influence QS.

Later research outcomes introduced contention, however. Anders and Chalemian (1974) questioned earlier conclusions about neonates after failing to replicate particular features of Emde et al.'s work (QS increase and SOL decrease, 1971). They were still able to replicate the increase in overall sleep time (measured across 3, 1-hr observations of eleven 3-day old infants following unanesthetized circumcision; along with Porter et al., 1999, excepting that in the 1999 study analgesics were occasionally used). It's worth noting that Emde et al. reported a total sleep time (TST) increase across a 10-hr interval following circumcision (1971), while the noncontiguous observations by Anders and Chalemian (1974) revealed that sleep pressure increases only after at least $1.5 \mathrm{hrs}$ following circumcision. A bout of increased wakefulness immediately following circumcision explained infants' additional sleep pressure, and their subsequent 'rebound' (1974), which is also noted to occur in sleep deprived adults. A single study in 1985 supported the overall sleep trends of Emde et al. (1971), but on terms more related to cortisol release and QS following unanesthetized circumcision. Their findings will be discussed in the upcoming section (Gunnar, Malone, Vance, \& Fisch, 1985). Others' results complemented those of 
Anders and Chalemian (Elander, Hellström, \& Qvarnström, 1993, who examined infants with inconsistent analgesic intervention). Otherwise, no further evaluation of circumcision's effect on sleep is apparent among the literature.

\section{Infant Nociceptive Responses and Sleep}

There is post-circumcision sleep recovery when analgesics are administered (Gedaly-Duff \& Huff-Slankard, 1998), showing that pain influences infant sleep quantity as well as quality. However, it's difficult to discern whether that change was the result of analgesic effects on sleep (Axelin, Kirjavainen, Salanterä, \& Lehtonen, 2010) or was an endogenously mediated response to pain relief, such as reduction in sympathetic nervous system (SNS) activation. Pain's activation of the SNS and the HypothalamicPituitary-Adrenal (HPA) endocrine response has a stimulating effect, so wakefulness might also be expected to increase as a result of cortisol production (Talbert, Kraybill, \& Potter, 1976; Gunnar et al., 1985). Once pain is relieved and cortisol is metabolized, infants may recover lost sleep. There is evidence of a return to baseline cortisol levels after only about 2.5hrs (1985), possibly explaining researchers' variation in QS outcomes during the post-circumcision period. Infant QS was reduced and their wakefulness was increased when their cortisol levels were high. While QS did increase after circumcision overall, it did not do so until cortisol levels were concomitantly reduced (1985). Sleep cycle data in the present study were obtained well beyond the $2.5 \mathrm{hr}$ window identified by Gunnar and colleagues. So, disproportionate wakefulness because of procedure-related cortisol expression isn't to be expected among these outcomes. If infants in the current sample experience rebound, or compensation, for lost QS following the immediate post-circumcision period, then the now equivocally-supported phenomenon should be observable in this study's sample.

Adults with rheumatologic disorders report easy arousability, possibly due to pain (Roehrs et al., 2013). If the unanesthetized surgery, and likely accompanying pain, increases infant sleep disturbance (as suggested by Anders and Chalemian's observations of increased wake in 1974; and Elander et al.'s observations of increased wake in 1993), then the transitional sleep of neonates in the present research 
would also be expected. If infants are potentiated to arouse from sleep following surgical intervention, then sleep fragmentation would also be expected to increase. The number of behavioral transitions, or frequent interruption of a contiguous sleep state, was used as a measure of fragmentation here.

Additionally, infants who may be experiencing pain from NPT during sleep would be expected to demonstrate more fragmented sleep, and particularly more fragmented AS. Although Emde et al. (1971) observed relative stability of AS proportion between circumcised and uncircumcised infants, they did not evaluate the quality of AS.

Alternative measures to help characterize pain during sleep include movement and respiratory rate (Beacham, 2004). Infants are fully capable of nociception (Ponder, 2002). Adverse tactile stimuli first elicit a withdrawal reaction, followed by a startle response, an increase in quiet sleep respiratory rate (QSRR), and sleep disruption, when presented to an infant during sleep (McNamara, Wulbrand, \& Thach 1998). Therefore, an increase in pain may also increase their number of startle responses during sleep. If the source of nociception is the surgical site, infants may not recoil, but the increase in respiratory rate should persist. The MMS recording provides respiratory output, and although some startle responses can be identified within the raw recording they are too transient to induce a change in sleep state scoring. Respiratory rate data during QS were, accordingly, interpreted to augment measures of architectural sleep disturbance. HPA-axis activation resulting from surgery or pain could reasonably also increase respiratory rate during sleep in the same way it might while awake. In a similar way, frequent sleep interruption accompanies SNS activity and could result in an increased sleep-related respiratory rate. Increases in QSrelated respiration, when the respiratory rate is typically at its lowest, appear to interfere with achieving developmental milestones at later ages (Montgomery-Downs \& Thoman, 1998). Pain sensation, then, may elicit any of the above reactions in neonates. 


\section{Statement of Problem}

Circumcision has the potential to interrupt neonatal sleep at a time when sleep may be adaptive for the infant. This may occur because of the procedure itself or because of associated pain. Its manifestation would result in acute sleep disturbance, with a particularly adverse effect on AS if pain is related to NPT. Data from motility monitoring of infant sleep can be used to quantify circumcision's effect on sleep episode architecture and quality. Similarly, physiological responses, like increased respiratory rate, can be measured in an effort to speculate on sleep disturbance or sleep-associated pain. The infant population studied is an ideal model for pain's effect on sleep because their circumcisions were unanesthetized. Evidence of sleep disturbance postoperatively has implications for both global use of anesthesia during circumcision and also for analgesic use in the U.S. Outcomes of research considering the circumcision-sleep relation could ultimately influence the practices of circumcision by medical professionals worldwide. To pursue this question of circumcision and sleep disturbance, sleep data (AS, QS, TS, and Latency to QS) and respiratory data from males circumcised without anesthesia and from uncircumcised infants (both male and female) were compared within and between groups using preexisting data.

\section{Methods}

\section{Participants}

Pregnant women were recruited from a small community hospital (Windham Memorial) in Willimantic, Connecticut from November of 1994 through October of 1999 to obtain the data being reevaluated presently. Only full-term ( $\geq 36$ weeks gestational age), low-risk infants were included in analyses. Infant medical records were obtained with parental consent, and mothers completed surveys for additional demographic information. Infants were excluded on the basis of maternal EtOH use, later diagnosed developmental disability, missing circumcision time/status, and (for respiratory analyses) respiratory intervention during recording. Not all data from the original study were available. Some data were missing because of technical recording error and some because information needed to identify infant 
age based on recorded data was unavailable. A description of the reduction from full sample size $(n=$ 124) to individual group samples is provided in Figure 2.

Circumcised infants. Circumcisions, performed at the discretion of the parents of male infants, were unanesthetized, which was typical practice at the time. Median latency to circumcision was $36 \mathrm{hrs}$ and $58 \mathrm{~min}(n=17)$. Post-circumcision data had a median latency of $11 \mathrm{hrs}$ and $49 \mathrm{~min}(n=18)$ from circumcision, and the median latency from birth to post-circumcision data was $30 \mathrm{hrs}$ and $22 \mathrm{~min}(n=18)$. Demographic information of three infant comparison groups is presented in Table 1, including maternal age and education level, 5-min APGAR score (Appearance, Pulse, Grimace, Activity, and Respiration), birthing and feeding methods, anesthetic administration during labor, and maternal parity. Specifically, infant feeding method (Oberlander, Barr, Young, \& Brian, 1992) and birthing method (Sadeh, Dark, \& Vohr, 1996; Freudigman \& Thoman, 1998) can influence early sleep organization. For comparisons in which infants differed in their distribution on these or other important demographic criteria, an additional comparison was used to verify that there were no infant differences based on the demographic criteria alone.

Control infants. Recruitment, screening, consent, and data collection for uncircumcised infants were similar to those of circumcised infants, excepting that female infants were also measured $(n=60 ; 40$ with repeated-measures data). The increased power that was afforded by female inclusion was not expected to affect sleep outcome measures, as neonatal sleep architecture does not differ based on sex alone through 43 weeks' conceptional age (Holditch-Davis, Scher, Schwartz, \& Hudson-Barr, 2004). However, because similar sleep architecture across sexes was based on longer-term development than is considered here, a subset of the male-and-female uncircumcised control group consisting of only uncircumcised males ( $n=15,6$ in repeated-measures analysis) was also used. Careful sex-based consideration was also given to QSRR measures, because of sex differences observed by the second day following birth (Montgomery-Downs \& Thoman, 1998). 


\section{Procedure}

The procedure for infant monitoring has been described elsewhere (Montgomery-Downs and Thoman, 1998). Briefly, infant movement and respiratory data were collected whenever infants were placed into a pressure-sensitive basinet during their hospital stay. In anticipation of this, a research team member was notified when consenting pregnant mothers were in labor so that recording could begin prior to delivery.

Raw data were first computer-scored based on a pre-existing algorithm developed and validated using infant sleep data, then verified by a research team member (Thoman \& Glazier, 1987). Data from this sample of infants were not obtained in anticipation of circumcision comparison. Therefore, researchers performing validity checks were unbiased and blinding to circumcision condition was not a concern. Recordings were synchronized to real-time during acquisition, and this information was used to match data with infant age.

\section{Measures}

The Motility Monitoring System is a nonintrusive apparatus used to collect macro-level behavioral data from sleeping infants. It minimally interferes with natural infant sleep regulation because instrumentation of infants is not necessary, unlike electroencephalography. Rather, the system consists of a thin, pressure-sensitive mattress that simultaneously records respiration and movement as one output trace (with movement overlapping respiration; Thoman \& Tynan, 1976). Infant sleep state is then interpreted based on respiratory pattern (which is distinctively different for active and quiet sleep) and movement (which is more typical of active sleep). It is both a reliable and valid system for quantifying infant active sleep, quiet sleep, and wake states (Thoman \& Tynan, 1979). It also allows for recording of infant behavior over an extended duration so long as the infant is in the crib.

QS, AS, Wake, and Out of Crib designations were applied to each 30sec segment (epoch) of data based on an algorithm (Thoman \& Glazier, 1987). Data scoring was reviewed by trained research team members. The present analysis evaluated these semi-raw data to identify qualifying sleep cycles. The average percentage of each sleep state (AS, QS, and TS), and the latency from AS at sleep cycle onset to 
QS later in the same sleep cycle, were compared between infant groups. Use of infant sleep cycle proportions, rather than overall proportion of sleep time, was a novel approach in this study compared to others using MMS. Inclusion of data in this reanalysis was contingent upon successful recording of at least one full, uninterrupted sleep cycle for which onset was observed, lasting a minimum of 30min. Sleep cycles were identified as one continuous bout of sleep that was comprised on one AS episode and one QS episode. To qualify, sleep cycles had to be uninterrupted by Out of Crib time, and must have been followed by more sleep data, rather than wake. These criteria were applied to reduce the likelihood that environmental disturbance affected the infant's sleep organization. Exclusion of infants based on cycle criteria is presented in Figure 2.

The index (quantity over a consistent interval of sleep) of TS was calculated by correcting the number of 30sec TS epochs for the hour-duration of a sleep cycle. For example, if a sleep cycle lasted for 30min and had one 30sec segment of TS, then the TS Index for that cycle equals 2 (\#TS epochs * (60/cycle duration)). Similar computation of TS Index was performed specifically using AS and QS duration within a cycle. The standardized outcome represents the frequency of a particular state per hour of sleep time.

AS, QS, and TS were quantified by their proportion within sleep cycles prior use in comparisons. Specifically, the number of $30 \mathrm{sec}$ epochs of each state was divided by the total number of sleep epochs observed in one full sleep cycle. This produced a percentage describing the amount of each state within a sleep cycle, standardized for sleep cycle durations. If an infant was observed for several full sleep cycles, then the mean percentage of each state was calculated to represent proportionality for that participant. There were no differences in the average number of cycles contributing to sleep state calculations between infant groups. Each infant's latency to QS was calculated by the minutes of sleep from onset of a cycle to the first epoch of QS in the same cycle. This excluded infants who only had cycles in the opposite order (QS-to-AS) from analysis. Infants observed for more than one cycle were represented by the average latency in all their cycles. 
Quiet sleep respiratory rate was quantified by researchers conducting the initial study of respiration and developmental outcomes (Montgomery-Downs \& Thoman, 1998). The number of breaths in $30 \mathrm{sec}$ of sleep time were counted, then multiplied by 2 to arrive at breaths per minute (Br/Min). The calculation outcome was used to characterize respiration during the 2 min interval from which the 30 sec sample was taken. Any $30 \mathrm{sec}$ sample during the $2 \mathrm{~min}$ could be used to define respiration for the entire 2 min interval. If an infant changed sleep state, moved, or experienced abnormal respiration (sighs or apneas) during the $30 \mathrm{sec}$ sample, then an alternate $30 \mathrm{sec}$ within the $2 \mathrm{~min}$ interval was selected to represent respiratory rate. In cases where movement artifact obliterated the respiratory signal for over $2 \mathrm{~min}$, researchers instead used the respiratory rate that was assigned to the previous $2 \mathrm{~min}$ of respiratory data rather than imputing the unreadable portion.

\section{Data Analysis}

Data were evaluated to ensure that assumptions for each comparison type were fulfilled (outliers, normality of distributions, and homogeneity of variance), and corrections for violations are noted in the results of individual analyses. A Dunn-Sidak corrected significance cutoff of .0253 was applied for $t$-tests of dependent means, of independent means, and for omnibus ANOVAs to define statistical significance. This conservative approach accounts for the fact that data from circumcised males were tested twice; once in comparison to a control group of both females and uncircumcised males, and again in comparison to a subset of that control group comprised only of uncircumcised males. Dunn-Sidak is a less conservative approach than Bonferroni that avoids over-correction for multiple tests using the same data. The difference in correction in this instance is small (versus .025), but is more precise. . An adjusted confidence level to ensure that familywise alpha was maintained at a maximum of .0253 was applied for all post-hoc pairwise comparisons, contingent upon rejection of the omnibus null Conventions for effect size (Cohen's $d$ ) were: small $\geq 0.2$, medium $\geq 0.5$, and large $\geq 0.8$. ANOVA effect sizes were calculated as Partial $\eta^{2}$, with conventions of: small $\geq 0.01$, medium $\geq 0.06$, and large $\geq 0.14$ (Aron, Aron, \& Coups, 2006). Descriptive statistics for demographic information are available in Table 1. Specific sample size for each group used in comparisons is available in Table 2. Effect sizes and post-hoc power (calculated 
using G*Power software) are also provided for tests with marginally significant outcomes or for which there were small sample sizes (both in results and in Tables 3-4).

Data were separated based upon infant circumcision status at the time of collection, generating two main infant groups and one sub-group: circumcised males $(n=17)$, both uncircumcised males and females $(n=40)$, and uncircumcised males alone from the larger male-and-female group $(n=6)$. Repeated-measures analyses were performed for each infant group for the variables in question. Data collected after circumcision (of circumcised males; $n=18$ ) were compared to time-matched data from uncircumcised infants $(n=60)$. Because the between-group comparison outcome may have resulted from sex differences, an additional comparison between only circumcised and uncircumcised males $(n=15)$ was performed. The sample size of uncircumcised males was not as large as anticipated because there were few uncircumcised male participants in general, and because few males were circumcised more than 24hrs after birth (rendering their data post-circumcision).

Research Question \#2, Exploratory Question \#2, was collapsed into the Mixed 2x2 RepeatedMeasures ANOVA and the One-Way ANOVAs (between groups) used for transitional index comparisons. This decision was based on a lack of variability (almost all infants scored 0 ) in the "movement" score during AS and in the "transitional" score during QS, which precluded utility of these measures in individual ANOVAs between state-specific index. "Movement" data scored during QS reflected an alternative coding in MMS data for TS, rather than representing a unique state on its own (as noted by the fact that QS generally has very little movement, and that startle responses are transient, above). As such, movement epochs were included in the proportion of TS calculations as well.

\section{Results}

\section{Research Question \#1: Does Organization of Sleep Episodes Following Unanesthetized Circumcision Deviate from Typical Infant Sleep Episodes?}


Hypothesis 1a: Transitional Sleep (TS) time, or Indeterminate Sleep (IS), will increase proportionate to total sleep time for circumcised infants, and will be higher than the IS proportion of uncircumcised infants.

\section{Exploratory Question 1a: Will the proportion of Active Sleep (AS) and of Quiet Sleep (QS) by infants after circumcision differ from those of uncircumcised (control) infants, or from sleep proportions prior to circumcision?}

Hypothesis 1a and Exploratory Question 1a are addressed simultaneously for each infant group (circumcised males, uncircumcised males and females, and uncircumcised males alone) because of the similarity in their analysis approach and because they involve the same samples of infants. Data from 57 infants were used in repeated measures analyses for AS, QS, and TS, and data from 78 infants were used in between-groups post-circumcision comparisons of AS, QS, and TS. There were no pre-existing differences in $\mathrm{AS} \%$, QS\%, or TS\% between circumcised and uncircumcised (male-and-female) groups. The average latency of sleep cycles from birth did not significantly differ between groups; neither did the average number of cycles contributing to measures of sleep proportion differ.

Repeated-measures $t$-tests were used to discern changes in the average percentage of behavioral states that compose sleep cycles: Active Sleep, Quiet Sleep, and Transitional Sleep. Table 1 describes characteristics of infants in the AS/QS/TS repeated measures sample and of infants in the Latency to QS sample based on delivery and feeding method, APGAR score at 5min, and maternal opioid analgesic, smoking, age, and parity. For circumcised males, cycles were defined as pre- or post-circumcision based on their own circumcision time, while for uncircumcised males and females, sleep cycles were designated as pre-matched or post-matched according to the average circumcision time of the circumcised male group (Median $=18 \mathrm{hr} 29 \mathrm{~min}, S D=6 \mathrm{hr} 26 \mathrm{~min}$ ). Data used in repeated-measures analyses were collected from infants between 1.73 and $64.75 \mathrm{hrs}$ after birth and from $29.82 \mathrm{hrs}$ preceding to $39.92 \mathrm{hrs}$ following circumcision. There was no difference in the number of sleep cycles contributing to infant averages preor post-circumcision. $T$-tests for independent means were used to evaluate pre-existing differences in AS, 
QS, and TS between circumcised males and the control (male and female) group from repeated-measures analyses. No pre-existing differences were present between groups $(p=.937$ for AS, $p=.688$ for QS, and $p=.329$ for TS).

Circumcised male repeated-measures test. The data from circumcised males $(n=17)$ were normally distributed for all three sleep parameters and no outliers were observed (cutoff z-score of 3.2 for skew, kurtosis, and outliers). QS percentage decreased from pre- to post-circumcision, $(p=.010$, Figure 3). TS percentage marginally increased from pre- to post-circumcision ( $p=.027$, Figure 4$)$. No change in AS percentage was observed $(p=.160)$. A post-hoc power analysis (2-tailed setting using G*Power software) revealed that the TS comparison was underpowered, and that with the same effect size an $n$ of 35 would be necessary to achieve $95 \%$ power. Table 2 presents repeated-measure comparison outcomes. Conservatively, the hypothesis that TS time would increase proportionate to total sleep in a cycle for circumcised infants was marginally supported, but consideration should be given to the medium-large effect size outcome. Although the average proportion of circumcised males' AS did not change across measurements, the average proportion of QS did decrease, in accordance with Exploratory Question 1a.

Tests between circumcised and uncircumcised infants. In a test comparing circumcised males' post-circumcision data to uncircumcised infants' (males and females, $n=60$ ) post-matched data, the circumcised male sample size increased from 17 to 18 infants because one infant with post-circumcision only data was included (see Table 1). Data used in analyses between groups were collected from infants between 18.17 and $64.75 \mathrm{hrs}$ after birth and from $29.82 \mathrm{hrs}$ preceding to $39.92 \mathrm{hrs}$ following circumcision (for circumcised males). There was no statistical difference in the latency from birth between any of the groups compared (Circumcised Males' $M=29 \mathrm{hr} 12 \mathrm{~min}$, Uncircumcised Males and Females' $M=28 \mathrm{hr}$ $35 \mathrm{~min}$, and Uncircumcised Males' $M=28 \mathrm{hr} 39 \mathrm{~min}$ ). Furthermore, there was no difference in the number of sleep cycles contributing to infant averages between circumcision groups. A Dunn-Sidak corrected significance cutoff of .0253 was used (see above). Active sleep proportion at the post-measurement had a large positive skew for the male and female control group and exceeded the 3.2 z-score cutoff. To accommodate test assumptions, groups' AS scores were square-root transformed prior to analysis. All 
comparisons met equality of variance assumptions (non-significant Levene's test).Circumcised males did not differ from post-matched data of uncircumcised infants (males and females) on any of the proportion sleep parameters after circumcision ( $p=.149, .079$, and .989 for AS, QS, and TS respectively; see Table 3 and Figures $3 \& 4$ ). Thus, the TS proportion of circumcised males was not higher than the TS proportion of uncircumcised infants, as anticipated. There was also no statistical difference in AS proportion between circumcised and control infants, and the decrease in QS proportion observed in the repeated-measures test did not create a meaningful difference when considered between the circumcision groups. A post-hoc power analysis was used to show that with similar proportions of infants between groups, very large $n$ 's ( 87 for uncircumcised males and 259 for circumcised infants) would be necessary to show significant changes in QS proportion in this comparison.

Control group repeated-measures tests. Females and uncircumcised males with both pre- and post-matched data $(n=40)$ were also considered across a similar period as circumcised males to aid in interpretation of the main outcomes. One infant was excluded from the analysis because of high outlying scores on multiple measures (AS and QS, cutoff z-score of 3.2). Data were normally distributed once this outlier was removed (cutoff z-score of 3.2 for skew and kurtosis). This uncircumcised group (males and females) did not evidence changes in the proportion of any sleep state across the time-matched period ( $p$ $=.585$ for AS, $p=.883$ for QS, Figure 3 , and $p=.212$ for TS, Figure 4$)$. Uncircumcised males alone $(n=$ 6) also maintained the proportion of each state ( $p=.237, .114, .366$ for AS, QS, and TS respectively) and did not differ from circumcised males at the post-matched measure (Table 3), although the sample size severely restricted power in these comparisons. A post-hoc power analysis showed that for the largest effect sizes (QS and TS) in repeated-measures analysis, power of just 15\% and 11\% was reached. For QS 41 infants, and for TS 58 infants, would be necessary to achieve $95 \%$ power in the repeated-measures analysis of uncircumcised males if the same effect sizes were observed.

Circumcision groups did not differ in maternal age or 5min APGAR score, and there were no differences in the distribution of maternal opioid analgesics or feeding method. Significantly more uncircumcised infants were Caesarean-delivered (Fisher's Exact Test, $p=.026$ ), but there were no 
differences in $\% \mathrm{QS}$ or $\% \mathrm{AS}$ based on delivery method alone. A difference in $\% \mathrm{TS}$ based on delivery method alone approached significance $(p=.041)$, possibly contributing to the lack of difference between infant groups at the post-circumcision measure; however, delivery could not account for the \%TS increase within circumcised males because all 17 infants were delivered vaginally.

\section{Exploratory Question 1b: Does QS latency from AS onset differ when circumcised infants} are compared to control infants, or when sleep preceding circumcision is compared to the sleep that

\section{follows?}

Data from 52 infants were used in repeated measures analyses for QS latency, and data from 73 infants were used to compare post-circumcision and post-matched QS latency (for characteristics, see Table 1). There are slight differences between the samples of infants used in the QS latency comparison because of its additional criterion that cycles included in the analysis begin with AS and end with QS. As a result, some infants with only QS-to-AS data at either pre-circumcision/matched or postcircumcision/matched measurements were disqualified from the analysis. Pre- and post-matched sleep cycles were determined as outlined above, using the new median circumcision time from the adjusted circumcised male group (adjusted average circumcision latency Median $=18 \mathrm{hr} 32 \mathrm{~min}, S D=6 \mathrm{hr} 38 \mathrm{~min}$ ).

There were no pre-existing differences in latency to QS between the groups. Neither the average latency of sleep cycles from birth nor the number of cycles contributing to latency differed between groups. No differences in maternal opioid analgesic, education, smoking during pregnancy, age, or parity were observed between the circumcised and uncircumcised infant groups for repeated-measures or independent-means comparisons of QS latency. There were also no differences in feeding method or APGAR score at 5min, although because all uncircumcised males had an APGAR score of 9 at $5 \mathrm{~min}$ it precluded their comparison to other groups as a continuous measure. Still, by considering mean scores, the uncircumcised males' average of 9 was very similar to the circumcised males' average score of 9.38. Although significantly more of the uncircumcised infants were Caesarean-delivered (Fisher's Exact Test, 
$p=.023)$, no differences in QS latency in either pre- or post-matched cycles were observed based on delivery method alone ( $p>.05$, Welch-Satterthwaite method for unequal variances).

Circumcised male repeated-measures test. The average latency from AS to QS in cycles was evaluated using a repeated-measures $t$-test, with one circumcised male infant excluded because only Quiet-to-Active state data were available after his circumcision $(n=16$, see Table 1$)$. The distribution of QS latency prior to circumcision was leptokurtic, and required a square root transformation. No change in A-Q latency was observed for the circumcised males ( $p=.333$, see Table 2 and Figure 5). Reflecting back on Exploratory Question 1b, the average latency to quiet sleep did not change from pre-circumcision to post-circumcision.

Tests between circumcised and uncircumcised infants. In a test between circumcised males with post-circumcision data and uncircumcised infants (males and females) with post-matched data, sample size differed: more infants were included because of qualifying data at only the post-measure, including one circumcised male $(n=17)$, while four female infants had only QS-to-AS cycles at pre/post-matched sleep measures and so were excluded from the analysis $(n=56$; see Table 1$)$. There was no difference in QS latency at the post-measure between groups in a $t$-test comparison of independent means (WelchSatterthwaite method for unequal variances, $p=.054$; see Table 3 and Figure 5). Although not statistically significant, the effect size of the latency comparison was large $(d=0.76)$. A post-hoc power analysis indicated that the comparison was underpowered (post-hoc power $=68 \%$ ), and that an increase in Ns to 36 and 106 (maintaining proportions of group membership) would have supplied power at 95\%. Conservatively, circumcised and uncircumcised (both male and female) groups did not differ in QS latency.

Control group repeated-measures tests. Similar to circumcised males, there was not a significant change in the latency to QS for uncircumcised males and females $(n=36, p=.717$; see Table 2 and Figure 5). Among six uncircumcised males alone, an increase in QS latency from pre-matched to postmatched data was statistically significant $(p=.021, d=0.89)$. No outliers existed to drive this outcome, and distributions met assumptions of normality in spite of their small size (z-cutoff of 3.2). For clarity, 
comparisons between circumcised males' post-circumcision data and the uncircumcised male sub-group's post-matched data were performed. Uncircumcised males with post-matched data included both males who were never circumcised and males not included in the repeated-measures analyses who had not yet been circumcised ( $n=15$, see Table 1$)$. There were, however, no significant differences in the postmeasure comparisons between male infants only $(p=.714$, see Table 3$)$.

Hypothesis 1b: The index of behavioral transitions will be higher for circumcised infants during sleep than for uncircumcised infants during sleep, and AS transitions will be more frequent overall compared to the uncircumcised control group. The circumcised infants' transitions will also be more frequent than they were prior to the procedure. More specifically, transitions during AS will be more frequent than transitions during QS for circumcised infants in both between- and within- groups (mixed) comparisons.

A Mixed 2x2 ANOVA was performed for the transitional indices calculated overall, during AS, and during QS each, with a repeated-measures factor of time and a fixed factor of circumcision group (Uncircumcised $\mathrm{N}=6$, Circumcised $\mathrm{N}=17$; see Table 1). No outliers were observed on any of the three measures for either circumcision group, and distributions of each measure were compliant with assumptions of normality (z-score, skew, and kurtosis cutoffs of 3.2). Box's M test was non-significant for unequal variances/covariances in all cases $(p>.001)$. Collinearity diagnostics indicated a lower than preferable tolerance, but a within-range Variance Inflation Factor; these measures are designed to be considered together, and some collinearity is to be expected in a partially repeated-measures design. Maximum Mahalanobis distance did not exceed the Chi-square cutoff $(p>.001)$ for any of the three comparisons $\left(\chi^{2}(2)<13.81\right)$ indicating no multivariate outliers. An initial alpha cutoff of .0253 was applied for the omnibus test (although specific a priori prediction of an interaction outcome was posed) because data used in the Mixed ANOVA overlapped with data used in One-way ANOVAs between groups. A Bonferroni correction of alpha based on four follow-up tests was applied for post-hoc comparisons. Although it is highly conservative, Bonferroni was selected rather than the Dunn-Sidak 
method applied in previous comparisons because the transition indices during AS and QS are dependent upon the overall transition index. Dunn-Sidak corrections are only permissible when the outcome measures of multiple tests can be considered independent of one another. Post-hoc pairwise power analyses were performed using a 2-tailed setting ( $\mathrm{G}^{*}$ Power software).

There were no pre-existing differences between circumcision groups. There also were no differences in the number of sleep cycles contributing to transition data. No difference in the latency to sleep measures from birth, in maternal age, in APGAR score at 5min, or in feeding method distributions existed. There were notably more Caesarean-delivered infants in the uncircumcised group than there were in the circumcised group of males, but infants did not differ in any of the transitional sleep measures based on delivery method alone $(p=.725, .111$, and .260 for overall transitions, transitions in AS, and transitions in QS respectively).

\section{Repeated-measure (Mixed) tests between circumcised and uncircumcised males. Mixed}

ANOVA outcomes and effect sizes are summarized in Table 2 and presented in Figures 6-8. In a comparison of circumcision group (between) and status (within) effects on overall transitional index during sleep cycles, there was a significant change from pre- to post-measures $(F(1,21)=7.75, p=.011)$. Pairwise comparisons need not follow because only two time groups were compared (pre and post). No effect of circumcision group beyond time was observed, as indicated by a non-significant time* circumcision interaction $(p=.853)$. Post-hoc comparisons using Bonferroni corrected alpha of .006 (accounting for 4 comparisons) revealed a marginally significant increase in the overall sleep transition index among circumcised males $(p=.007)$ and a significant increase in the sleep transition index during AS among circumcised males $(p=.001)$ from pre-circumcision to post-circumcision. Uncircumcised males did not have statistically significant outcomes for either overall transition index change $(p=.259)$ or for transition index during AS change $(p=.280)$.

The small sample size of uncircumcised males severely restricted power in evaluating overall and AS transitional index changes ( $4 \%$ and $5 \%$ post-hoc power, respectively). With the medium-large effect size outcomes of these comparisons maintained, a sample size of 47 for overall transitions and 66 for AS 
transitions would have delivered $95 \%$ power. Taken together, these outcomes indicate that the increase observed in transitional index overall among circumcised infants is likely accounted for by the increase in their behavioral transitions during AS. Transitional index overall and during AS increased following circumcision as hypothesized. Although the uncircumcised males evidenced a similar trend from prematched to post-matched data, effect sizes were not comparable in magnitude to those of the circumcised group and would have required a very large control sample (at least 176\% larger than the $n=17$ of the circumcised group) to provide the power necessary for statistical significance.

Tests (ANOVA) between circumcised and uncircumcised males. To further explain the increase in behavioral transitions during AS, three One-way ANOVAs were used to evaluate differences between circumcised males $(n=18)$ and the sub-group of uncircumcised males $(n=15$, see Table 1$)$. Recall that the comparisons of post-circumcision/matched data included additional uncircumcised males whose data were obtained prior to their own circumcision. Omnibus tests in each of the ANOVAs were interpreted as $t$-test outcomes because only two groups were compared. The same outliers identified in previous between-groups measures were initially excluded (one multivariate outlier for AS and QS proportions and one outlier for the number of cycles contributing to data averages). No additional univariate outliers were observed for any of the three transition-related post-circumcision measures in either circumcision group. Unequal variances between circumcised and uncircumcised males' transitional index during QS demanded an inverse transformation to satisfy test assumptions. ANOVA is robust to this violation when sample sizes are similar, as is the case in this comparison, so test outcomes were similar with and without transformation. An alpha cutoff of .0253 was used in analyses (see above).

None of the pairwise comparisons (transition index overall, transitions in AS, and transitions in QS) had a statistically significant outcome between male-only circumcision groups at the postmeasurement $(p=.891, .761$, and .738 for overall, during AS, and during QS, respectively; see Table 3 and Figures 7-8). Despite low power outcomes for these ANOVAs in post-hoc analysis, effect sizes were also very small. Unreasonably large sample sizes would have been required to reveal differences between groups (over 2000 infants in each group, using $\mathrm{G}^{*}$ Power for post-hoc power analyses). 


\section{Research Question \#2: Does Sleep Following Unanesthetized Circumcision Reflect Acute Nociception?}

Hypothesis 2: Infants who underwent unanesthetized circumcision will have a higher respiratory rate during QS than uncircumcised male infants. Circumcised male infants will have a higher respiratory rate during sleep than they had prior to their circumcision.

Quiet sleep respiratory rate (QSRR) was compared across circumcision or time-matched measures and also between infants' post-circumcision/matched data $(n=46)$ to determine evidence of increased sympathetic tone during sleep. QSRR (breaths/min) samples from the first, middle, or last $5 \mathrm{~min}$ periods of each QS bout when the infant was in the crib were averaged to obtain overall QSRR preceding and following circumcision. One circumcised male infant was excluded from respiratory analyses because of a uniquely elevated respiratory rate that resulted in clinical intervention and one was excluded because of maternal EtOH use. The 16 remaining circumcised males with qualifying pre- and post- QSRR data had an mean circumcision time of $16.79 \mathrm{hrs}$ after birth $(S D=8.42 \mathrm{hrs})$ and all were vaginally delivered. The mean circumcision time of males was used as a cutoff to match data from uncircumcised infants to pre- and post-circumcision data. Other demographic distributions are presented in Table 1. A significance cutoff of .0253 was used (see above).

There were no pre-existing differences in QSRR between infant groups. Infants did not significantly differ in their latency to respiratory measures from birth. The average respiratory rate of circumcised males was log-transformed for repeated-measures analysis, and all respiratory rates were logtransformed for analyses between groups. There were no differences in the number of RR samples from early, middle, or late QS periods (Montgomery-Downs \& Thoman, 1998) used in analyses. There were also no pre-existing differences in RR between groups. Infant groups did not differ in APGAR score at $5 \mathrm{~min}$, in maternal age, or in the distribution of maternal opioid analgesics, feeding, or birthing method.

Circumcised male repeated-measures test. Male infants with both pre- and post-circumcision data $(n=16$, see Table 1$)$ were considered for their change across the two measures. There were no outlying infants based on average QSRR. A logarithmic transformation of pre- and post-circumcision data 
was used to correct a leptokurtic distribution (exceeding 3.2 times standard error) at post-circumcision. Pre-circumcision data had a negative skew, and so prior to log-transform these data were transformed to a positive distribution (using (1+highest)-score). QSRR significantly increased when average respiration prior to circumcision was compared to that after circumcision in the repeated measures analysis $(p=.006$, see Figure 9). Circumcised infants demonstrated a statistically significant increase in QSRR, as predicted.

Tests between circumcised and uncircumcised infants. Infants were then compared between the post-circumcision and post-matched measures to evaluate how similar the outcomes of their QSRR profiles were. Sample size in the between-groups comparison $(n=58 ; 22$ males and 36 females, 17 circumcised and 41 uncircumcised) was larger than in repeated-measures comparisons because infants with only post-circumcision (or post-matched) data were included. A similar log-transformation as above was applied to accommodate distribution assumptions. There were no differences in QSRR between infant groups ( $p=.162$, see Table 3 and Figure 9). A post-hoc power analysis ( $\mathrm{G}^{*}$ Power software) revealed that achieving 95\% power would have required 113 and 227 infants (with similar group membership ratio) if a similar effect size were observed in this comparison. An additional comparison between circumcised and uncircumcised male infants only was under-powered and demonstrated no group differences $(p=.254)$. In spite of a medium-large effect size, the required sample sizes to achieve 95\% power are very large (195 and 49).

Control group repeated-measures tests. To aid in interpretation of consistent QSRR among circumcised males, a comparison group of uncircumcised infants composed of 4 uncircumcised males and 26 females, of whom one was Caesarean-delivered, was considered. No differences in feeding method, birth method, or 5-min APGAR score, and no differences in maternal parity, opioid analgesic during labor, education, age, or smoking during pregnancy were observed between circumcised and uncircumcised infant groups.

Circumcised infants' data had a leptokurtic distribution, so data were log-transformed prior to comparison to meet distribution assumptions. An increase in average QSRR was observed from prematched to post-matched samples across the uncircumcised (male and female) control group $(p=.010$, 
see Table 2), that was similar in direction and magnitude to the circumcised group. Although low in power, an additional repeated-measures comparison for uncircumcised males only was performed $(n=4)$ to consider effect size magnitude $(p=.792)$.

\section{Discussion}

\section{Summary of Outcomes}

The circumcised male group appears to have experienced changes in QS and TS that differed from the female and uncircumcised groups' trends. Their decrease in QS and increase in TS cannot be unequivocally attributed to circumcision status of males because limited power in the uncircumcised male group prevented ruling-out a sex difference. There was also ultimately no difference in TS proportion between circumcised males and control infants.

While there was a meaningful increase in QS latency for uncircumcised males, it did not ultimately extend to a difference between the circumcised and uncircumcised male groups. Circumcised males experienced a similar QS latency before and after circumcision. From a conservative perspective, similar QS latency was experienced between the larger (male and female) control group and the group of circumcised males. This is because the test outcome did not reach statistical significance at the very restrictive .0253 alpha cutoff. However, these groups may have differed in their post-measure QS latency with a larger sample in light of the medium effect size of their difference. No pre-existing difference between the circumcised and uncircumcised infant groups could explain this outcome. Because uncircumcised males had such a dramatic change in QS latency across measures while the combined group did not, and because uncircumcised males also had no pre-existing differences in QS latency compared to circumcised males in the repeated-measures groups, the possibility exists that circumcision subdued a normal increase in latency to QS for males across the first days following birth.

Circumcised and uncircumcised males appear to experience a similar number of behavioral transitions during overall sleep and also during active sleep. This is in contention with the apparent increase in transition index of circumcised males from pre-circumcision to post-circumcision, but a non- 
significant increase for the uncircumcised group at time-matched sleep measures. Therefore, the change in the number of behavioral transitions experienced by circumcised males, although meaningful within the group, did not ultimately create a difference between them and uncircumcised males. This, along with a lack of time*circumcision interaction in the Mixed ANOVA, indicates that transition index change was not heavily influenced by circumcision status.

Circumcised infants demonstrated a similar change in QSRR as uncircumcised infants. In a between-groups comparison of post-circumcision and post-matched data, no meaningful difference in QSRR was observed. Most infants demonstrated an increase in QSRR regardless of circumcision status or sex. The hypothesis that circumcised infants would have a higher QSRR than uncircumcised infants because of increased sympathetic tone was not supported.

\section{Interpretation of Outcomes}

The present analyses showed there were significant decreases in QS proportion within sleep cycles from pre-circumcision to post-circumcision. The same outcome was not observed in either control group of infants. Gunnar and colleagues (1985) also noted no significant change in the percentage of time spent in QS (not restricted to sleep cycles) in a sample of $13,<2$ day old, uncircumcised male infants across $2.5 \mathrm{hrs}$ of observation. A decrease in sleep, including QS, following circumcision has been reported by Anders and Chalemian (1974) among eleven 3-day old infants, but their outcome and ours contend with data from several other studies considering changes across circumcision, stressors, and within the first two days of life. One of the earliest studies by Emde et al. (1971) found evidence of increases in QS among a small sample $(n=10)$ of circumcised infants, half of whom had a concomitant increase in AS. Increases in QS proportion also occur from the day of, to the day following, birth across species (including a sample of 47 humans; Carroll, Denenburg, \& Thoman, 1998). Shifts in QS following circumcision appear to be inversely related to plasma cortisol levels (Gunnar et al., 1985), which are known to increase dramatically following unanesthetized circumcision (Gunnar et al., 1988), but no conclusive comparison was made concerning changes in QS itself across measurements or between circumcision groups in cortisol studies. The difference in QS outcome between this analysis and others 
deserves interpretation, as it may indicate a unique factor or difference in design that influences infant outcome.

There were also distinct differences in the latency to QS observed here in comparison to literature (Emde et al., 1971) and expected developmental outcomes (Groome, Swiber, Atterbury, Bentz, \& Holland, 1997). The sample of circumcised males in this study demonstrated no change in latency to QS, while Emde et al. reported notable decreases and suggested that this was indicative of QS propensity in circumcised infants (1971). Developmentally, latency from AS to QS has been shown to decrease from late fetal to 4-week postnatal measurements (Groome et al., 1997). Similarly, QS in infants progressively increases across the first few years of age (Korner, 1968). Still, sleep organization unique to the first few days of life is not an atypical proposal (Carroll, Denenburg, \& Thoman, 1998; Gunnar et al., 1988). Because no change in the latency to QS was observed here, as might be expected considering the QS decrease among circumcised males, some factor appears to be either truncating or replacing QS. This possibility is supported by the dramatic increase in QS latency seen in this study's small sample of uncircumcised males. If an initial increase in latency to QS within sleep cycles is a normal response to the stress of birth (Carroll et al., 1998), then the combined (male and female) control group should also have demonstrated increases, but this was not the case. The increase in QS latency, then, could be specific to males but mitigated among circumcised males (possibly because of circumcision itself), or could be the result of an anomalous sample of uncircumcised males considering its size.

One unique and potentially meaningful difference in measuring QS between the studies above and the present analysis is the use of sleep state proportions within qualifying sleep cycles. Rather than using the total time spent in QS during a bout of behavioral observation, only sleep cycles of a minimum duration (30min) and with specific qualifying criteria (only sleep could follow a qualifying cycle, and a cycle could not be interrupted by out-of-crib time) were used to generate the proportion of QS for infants in this study. The more stringent criteria applied for the first time here, although eliminating some data from analysis, allowed for more confidence in the observed differences' association with intrinsicallydriven infant sleep architecture. Cycle specificity was achieved while still accommodating a broader 
continuous recording interval through motility monitoring, as opposed to brief bouts of observation (Gunnar et al., 1985 and 1988). For example, Emde et al. (1971) recognized an increase in the number of QS episodes after circumcision that was robust enough to reveal a difference between circumcised $(n=$ $10)$ and uncircumcised males $(n=10)$. While this study did not evaluate the number of bouts of particular sleep states, the increase could account for differences in outcome: Latency to the first QS episode in their study could have decreased because unadulterated cycles were not parsed out from among the data for analysis, and could simply reflect sleep propensity for the first episode of QS alone instead of extrapolating to later sleep. In fact, taken together, their latency outcome and this study's carry interesting implications. Infants may have less QS within consolidated sleep cycles that, in turn, creates a propensity for more frequent QS bouts and longer non-contiguous QS episodes. Also, some research has not distinguished TS from other states in determining state changes (Emde et al., 1971), or has grouped TS in among other states rather than isolating it as a developmentally-meaningful state on its own (Anders \& Chalemian, 1974).

Increases in QS following circumcision or stressful events have been speculated to serve a conservation-withdrawal (Emde et al., 1971) purpose in the face of aversive stimuli that an infant cannot escape. Cortisol studies do not fuse well with this interpretation, although data have been superficially similar in terms of a later QS rebound, because QS is in fact minimal during elevated cortisol (Gunnar et al., 1985). There is no apparent purpose for a withdrawal response after cortisol has decreased, indicating the end of a stress response, especially considering the lapse between a stressor and a measureable cortisol increase (Porges, 1992). In relation to present outcomes, an initially strong decrease in QS presumably accompanying cortisol elevation is unlikely to have driven the decrease in QS observed. This is because of the extended period over which post-circumcision data were averaged here, and because evidence from Gunnar et al. (1985) indicates that cortisol increases following circumcision return to baseline within about four hours. It becomes plausible, then, that some extended type of adverse stimulus persists following the circumcision procedure that, while not pervasive enough to affect the infant's cortisol levels, may have an effect on sleep architecture via sleep fragmentation. One such reasonable 
stimulus is aggravation of the postoperative wound during nocturnal penile tumescence, which occurs rarely during wake but is ubiquitous during infant sleep, most prevalently during AS (Karacan, 1968; Korner, 1968, 1969). To ascertain the amount of fragmentation or disturbance during infant sleep cycles, transitional sleep proportion and the number of behavioral transitions were evaluated.

Increases in the proportion of transitional sleep were marginally significant among circumcised males and were not observed among control infants. Changes in transitional indices appeared to best explain that likely change in transitional sleep proportion: the index of transitions during AS increased for circumcised males, while the index during QS did not. This is interesting considering that no change in latency to QS was observed, and that trends for AS proportion (although not statistically significant) were increasing. A potential schematic demonstrating these combined changes in cycle organization for circumcised males is presented in Figure 10. No change in QS latency from AS, taken with an unchanged transition index during QS, suggests that the increase in behavioral transitions during AS observed in the circumcised group probably explains the increase in their overall transitional index (standardized against sleep cycle duration), and therefore in their proportion of transitional sleep.

While no change in transitional sleep index was observed among uncircumcised males and no time*circumcision interaction existed between the male groups, the power to detect these differences was low because of a limited uncircumcised sample size. The TS index change among uncircumcised males may have been statistically significant with universally greater power, but it is important to recognize that power is also influenced by effect size. Greater power through increased sample size would also have increased the sensitivity of the mixed ANOVA to detect the dramatic difference in transitional index during AS effect size between circumcised and uncircumcised infants Considering that the relation between sample size and power is based on effect size, it makes sense to interpret the ANOVA outcomes based on effect sizes from post-hoc pairwise comparisons.

Uncircumcised males had an increasing trend in transitional sleep index overall and during AS, and a decrease during QS, all of which were similar in direction to the circumcised male group. Their increase in transitions during AS, however, was notably smaller in effect size than the increase by 
circumcised males. Circumcised males appear to have a different transitional sleep change than uncircumcised infants because of that difference in magnitude. Male groups ultimately did not differ in their number of behavioral transitions even during AS, so even if circumcision status was the source of differences in their trends it is unlikely that its effect created meaningful sleep qualify differences between males. Still, the organization of TS is a valuable predictor of developmental outcome for infants (particularly when those transitions occur predominantly during AS), and increased TS is typical of premature, at-risk infants (Weisman, Magori-Cohen, Louzoun, Eidelman, \& Feldman, 2011). The increase observed in this research raises concern that circumcision may be related to shifts in transitional sleep time or behavioral transition frequency (sleep stability) specifically within AS, and deserves further investigation. Interestingly, and as observed in other research investigating changes in AS alone (Gunnar et al., 1985; Emde et al. 1971; Anders \& Chalemian, 1974), no change in AS proportion accompanied the marginal increase in TS or the increase in behavioral transitions during AS episodes. An appropriate conjecture would be that infants are sleeping more (having more sleep episodes) overall or are having longer sleep episodes to account for an increase in transitional sleep or behavioral transitions the same way that adults rebound from reduced REM.

Respiratory rate increased significantly from before to after as anticipated, but did not seem to reflect differences based on circumcision status. It was expected that an increase in sympathetic tone would result increase QSRR for circumcised infants, but the female and uncircumcised male control group of infants also experienced a QSRR increase. The groups' post-circumcision and post-matched data were also similar. An overall increase in QSRR among 62 neonates was concluded by MontgomeryDowns and Thoman (1998) in an analysis across the first two postnatal days, but they explicitly excluded circumcised infants from analyses. The increase in QSRR that they noted was lower for females $(n=36)$ than for uncircumcised males $(n=27)$ by day two. The post-circumcision comparison between circumcised males in this analysis and the male-and-female control group didn't evidence a significant difference in QSRR, but the consistent trends among the two larger groups were congruent with their (Montgomery-Downs and Thoman's, 1998) outcomes. 
This research met with several limitations during its progress, most notably adequate control group sample size and the expectation that infant movement was scored in isolation from transitional sleep rather than as its corollary during QS. The use of motility monitoring has been well validated, but the technique of isolating sleep cycles that meet duration and continuity criteria has not been vetted. This acknowledged, use of cycles delivers a strength: overall state proportion may change within all sleep, but architecture within cycles defines the unit of infant (and adult) sleep recognized in the field. If the two features differ, as appears to be the case when this study's outcomes are compared to outcomes from other work on early infant sleep, then future research might seek to clarify the cause of that disparity. There is also translational value in this analysis because there is no concrete evidence that contemporarily circumcised infants continue to receive analgesia at a duration following circumcision, but it appears that they still experience changes in sleep organization extending through at least the first day and a half following circumcision.

In conclusion, the change in QS proportion within sleep cycles appears to decrease following circumcision, which is contrary to observations in the literature of its increase in proportion relative to total sleep following circumcision. These outcomes deserve to be taken together in evaluation of infant sleep microarchitecture during the first few days following birth. Transitional sleep, small amounts of which are related to developmentally favorable outcomes, increased for circumcised males while it did not for other infant groups. The number of behavioral transitions also appeared to increase more for circumcised males than for uncircumcised males, which future work should seek to replicate. An increase in latency to QS within sleep cycles by uncircumcised males may represent a normal developmental trajectory that circumcision potentially interferes with, considering the lack of change among circumcised males. Work with uncircumcised groups should seek to clarify whether this study's small uncircumcised sample was anomalous in this feature, or whether the increase is a sex-based difference between males and females that is also altered by circumcision condition. The difference between reduced latency to the first bout of QS overall in other research is counter-intuitive compared to the stability within sleep cycles observed here, and begs further investigation. This research indicated that circumcised males appear to 
have different QS, TS, and possibly latency-to-QS profiles shortly after birth in comparison to control groups that are not rooted in pre-existing differences, but also indicates that their changes did not appear to define them as distinct from other groups on final post-circumcision measures. Their changes may be related to their circumcision status, but whether those changes create meaningful differences in outcome for the circumcised males remains to be answered. 


\section{References}

American Academy of Pediatrics (1999), Reaffirmed (2005). Circumcision policy statement. Pediatrics, 103(3), 686-796.

American Academy of Pediatrics (2012). Circumcision policy statement. Pediatrics, 130(3), 585-586.

Anand, K. J. (1998). Clinical importance of pain and stress in preterm neonates. Biology of the Neonate, 73(1), 1-9.

Anand, K. J., Brown, M. J., Causon, R. C., Christofides, N. D., Bloom, S. R., \& Aynsley-Green, A. (1985). Can the human neonate mount an endocrine and metabolic response to surgery? Journal of Pediatric Surgery, 20(1), 41-48.

Anand, K. J., \& Hickey, P. R. (1988). Pain and its effects in the human neonate and fetus. Journal of Prenatal \& Perinatal Psychology \& Health, 3(2), 103-123.

Anand, K. J., Johnston, C. C., Oberlander, T. F., Taddio, A., Lehr, V. T., \& Walco, G. A. (2005). Analgesia and local anesthesia during invasive procedures in the neonate. Clinical Therapeutics, 27(6), 844-876.

Anders, T.F. Organization and development of sleep in early life. Rev ed. Petit D., topic ed. In: Tremblay R. E., Boivin M., Peters R. D., eds. Encyclopedia on Early Childhood Development [online]. Montreal, Quebec: Centre of Excellence for Early Childhood Development and Strategic Knowledge Cluster on Early Child Development; 2010:1-8. Available at: http://www.childencyclopedia.com/documents/AndersANGxp2.pdf.

Anders, T. F., \& Chalemian, R. J. (1974). The effects of circumcision on sleep-wake states in human neonates. Psychosomatic Medicine, 36(2), 174-179.

Anders, T. F., \& Sostek, A. M. (1976). The use of time lapse video recording of sleep-wake behavior in human infants. Psychophysiology, 13(2), 155-158.

Aron, A., Aron, E. N., \& Coups, E. J. (2006). Statistics for Psychology (4 ${ }^{\text {th }}$ ed.). Upper Saddle River, NJ: Pearson Education, Inc. 
Axelin, A., Kirjavainen, J., Salanterä, S., \& Lehtonen, L. (2010). Effects of pain management on sleep in preterm infants. European Journal of Pain, 14(7), 752-758.

Beacham, P. S. (2004). Behavioral and physiological indicators of procedural and postoperative pain in high-risk infants. Journal of Obstetric, Gynecologic, and Neonatal Nursing, 33(2), 246-255.

Benini, F., Johnston, C. C., Faucher, D., \& Aranda, J. V. (1993). Topical anesthesia during circumcision in newborn infants. Journal of the American Medical Association, 270(7), 850-853.

Carroll, D. A., Denenberg, V. H., \& Thoman, E. B. (1999). A comparative study of quiet sleep, active sleep, and waking on the first 2 days of life. Developmental Psychobiology, 35(1), 43-48.

Dauvilliers, Y., \& Touchon, J. (2001). Sleep in fibromyalgia: Review of clinical and polysomnographic data. [Abstract]. Clinical Neurophysiology, 31(1), 18-33.

Dixon, S., Snyder, J., Holve, R., \& Bromberger, P. (1984). Behavioral effects of circumcision with and without anesthesia. Journal of Developmental and Behavioral Pediatrics, 5(5), 246-250.

Elander, G., Hellström, G., \& Qvarnström, B. (1993). Care of infants after major surgery: Observation of behavior and analgesic administration. Pediatric Nursing, 19(3), 221-226.

Emde, R. N., Harmon, R. J., Metcalf, D., Koenig, R. L., \& Wagonfeld, S. (1971). Stress and neonatal sleep. Psychosomatic Medicine, 33(6), 491-498.

Engel, G. L. (1962). Anxiety and depression-withdrawal: The primary effects of unpleasure. International Journal of Psychoanalysis, 43, 89-97.

Engel, G. L., \& Schmale, A. H. (1972). Conservation-withdrawal: A primary regulatory process for organismic homeostatis. In R. Porter \& J. Knight, (eds.), Physiology, emotion \& psychosomatic illness. New York, NY: Associated Scientific Publishers.

Everson, C. A., Bergmann, B. M., \& Rechtschaffen, A. (1989). Sleep deprivation in the rat: III. Total sleep deprivation. Sleep: Journal of Sleep Research \& Sleep Medicine, 12(1), 13-21.

Fifer, W. P., Byrd, D. L., Kaku, M., Eigsti, I., Isler, J. R., Grose-Fifer, J., Tarullo, A. R., \& Balsam, P. D. (2010). Newborn infants learn during sleep. PNAS Proceedings of the National Academy of Sciences of the United States of America, 107(22), doi:10.1073/pnas.1005061107 
Freudigman, K. A., \& Thoman, E. B. (1998). Infants' earliest sleep/wake organization differs as a function of delivery mode. Developmental Psychobiology, 32(4), 293-303.

Gedaly-Duff, V., \& Huff-Slankard, J. (1998). Sleep as an indicator for pain relief in an infant: a case study. Journal of Pediatric Nursing, 13(1), 32-40.

Geyer, J., Ellsbury, D., Kleiber, C., Litwiller, D., Hinton, A., \& Yankowitz, J. (2002). An evidence-based multidisciplinary protocol for neonatal circumcision pain management. Journal of Obstetric, Gynecologic, \& Neonatal Nursing, 31(4), 403-410.

Groome, L. J., Swiber, M. J., Atterbury, J. L, Bentz, L. S., \& Holland, S. B. (1997). Similarities and differences in behavioral state organization during sleep periods in the perinatal infant before and after birth. Child Development, 68(1), 1-11.

Grunau, R. E., Holsti, L., Whitfield, M. F., \& Ling, E. (2000). Are twitches, startles, and body movements pain indicators in extremely low birth weight infants? The Clinical Journal of Pain, $16(1), 37-45$.

Gunnar, M. R., Malone, S., Vance, G., \& Fisch, R. O. (1985). Coping with aversive stimulation in the neonatal period: Quiet sleep and plasma cortisol levels during recovery from circumcision. Child Development, 56(4), 824-834.

Gunnar, M. R., Porter, F. L., Wolf, C. M., Rigatuso, J., \& Larson M. C. (1995). Neonatal stress reactivity: Predictions to later emotional temperament. Child Development, 66, 1-13.

Herman, J. H. (2005). Chornobiology of sleep in children. In Sheldon, S.H., Ferber, R., and Kryger, M.H. (eds.), Principles and Practice of Pediatric Sleep Medicine. Maryland Heights, MO: Elsevier Saunders, Inc.

Holditch-Davis, D., Scher, M., Schwarts, T., \& Hudson-Barr, D. (2004). Sleeping and waking state development in preterm infants. Early Human Development, 80(1), 43-64.

Holsti, L., Grunau, R. E., Oberlander, T. F., Whitfield, M. F., \& Weinberg, J. (2005). Body movements: An important additional factor in discriminating pain from stress in preterm infants. The Clinical Journal of Pain, 21(6), 491-498. 
Karacan, I., Marans, A., Barnet, A., \& Lodge, A. (1968). Ontogeny of penile erection during sleep in infants. [Abstract]. Psychophysiology, 4(3), 363-364.

Kaufman, C., \& Rosenblum, L. (1967). The reaction to separation in infant monkeys: Anaclitic depression and conservation-withdrawal. Psychosomatic Medicine, 29(6), 648-675.

Korner, A. (1968). REM organization in neonates: Theoretical implications for development and the biological function of REM. Archives of General Psychiatry, 19, 330-340.

Korner, A. (1969). Neonatal startles, smiles, erections, and reflex sucks as related to state, sex, and individuality. Child Development, 40(4), 1039-1053.

Lagercrantz, H., \& Slotkin, T. A. (1986). The 'stress' of being born. Scientific American, 254(4), 100-107.

Lampl, M. \& Johnson, M. L. (2011). Infant growth in length follows prolonged sleep and increased naps. Sleep, 34(5), 641-650.

Lander, J., Brady-Fryer, B., Metcalfe, J. B., Nazarali, S., \& Muttitt, S. (1997). Comparison of ring block, dorsal penile nerve block, and topical anesthesia for neonatal circumcision: A randomized controlled trial. JAMA, 278(24), 2157-2162.

Lee, B. H. (2002). Managing pain in human neonates - applications for animals. Journal of the American Veterinary Medical Association, 221(2), 233-237.

Mathew, P. J., \& Mathew, J. L. (2003). Assessment and management of pain in infants. Postgraduate Medical Journal, 79, 438-443. Electronic, accessed from pmj.bmj.com on 29 October, 2012.

McNamara, F., Wulbrand, H., \& Thach, B. T. (1998). Characteristics of the infant arousal response. Journal of Applied Physiology, 85, 2314-2321.

Montgomery-Downs, H.E., \& Gozal, D. (2006). Sleep habits and risk factors for sleep-disordered breathing in infants and young toddlers in Louisville, Kentucky. Sleep Medicine, 7(3), 211-219.

Montgomery-Downs, H. E., \& Thoman, E. B. (1998). Biological and behavioral correlates of quiet sleep respiration rates in infants. Physiology \& Behavior, 64(5), 637-643. 
Morison, S. J., Holsti, L., Grunau, R. E., Whitfield, M. F., Oberlander, T. F., Chan, H. W. P., \& Williams, L. (2003). Are there developmentally distinct motor indicators of pain in preterm infants? Early Human Development, 72(2), 131-146.

Oberlander, T. F., Barr, R. G., Young, S. N., \& Brian, J. A. (1992). Short-term effects of feed composition on sleeping and crying in newborns. Pediatrics, 90(5), 733-740.

Oztürk, L., Pelin, Z., Karadeniz, D., Kaynak, H., Cakar, L., \& Gözükirmizi, E. (1999). Effects of 48 hours sleep deprivation on human immune profile. Sleep Research Online: SRO, 2(4), 107-111.

Peters, J. W. B., Koot, H. M., deBoer, J. B., Passchier, J., Bueno-de-Mesquita, J. M., deJong, F., H., Duivenvoorden, H. J., \& Tibboel, D. (2003). Major surgery within the first 3 months of life and subsequent biobehavioral pain responses to immunization at later age: A case comparison study. Pediatrics, 111(1), 129-135.

Piteo, A. M., Lushington, K., Roberts, R. M., Martin, A. J., Nettelbeck, T., Kohler, M. J., \& Kennedy, J. D. (2011). Parental-reported snoring from the first month of life and cognitive development at 12 months of age. Sleep Medicine, 12: 975 - 980.

Ponder, B. L. (2002). Effects of pain in the human neonate. American Journal of END Technology, 42, 210-223.

Porges, S. W. (1992). Vagal tone: A physiological marker of stress vulnerability. Pediatrics, 90(3), 498504.

Porter, F. L., Wolf, C. M., Gold, J., Lotsoff, D., \& Miller, J. P. (1997). Pain and pain management in newborn infants: A survey of physicians and nurses. Pediatrics, 100(4), 626-632.

Porter, F. L., Wolf, C. M., \& Miller, J. P. (1999). Procedural pain in newborn infants: the influence of intensity and development. Pediatrics, 104(1), e13, pediatrics.aappublications.org, accessed 29 October 2012.

Ramenghi, L. A., Wood, C. M., Griffith, G. C., \& Levene, M. I. (1996). Reduction of pain response in premature infants using intraoral sucrose. Archives of Disease in Childhood, 74(2), F126-F128. 
Roehrs, T., Diederichs, C., Gillis, M., Burger, A. J., Stout, R. A., Lumley, M. A., \& Roth, T. (2013). Nocturnal sleep, daytime sleepiness and fatigue in fibromyalgia patients compared to rheumatoid arthritis patients and healthy controls: a preliminary study. Sleep Medicine, 4(1), 109-115.

Sadeh, A., Dark, I., \& Vohr, B. R. (1996). Newborns' sleep-wake patterns: The role of maternal, delivery and infant factors. Early Human Development, 44(2), 113-126.

Sheldon, S. H. (2005). Polysomnography in infants and children. In Sheldon, S.H., Ferber, R., and Kryger, M.H. (eds.), Principles and practice of pediatric sleep medicine. Maryland Heights, MO: Elsevier Saunders, Inc.

Sitka (1990). The startle reaction of the newborn infant. [Abstract]. Zentralbl Gynakol, 112(14), 911-919.

Slater, R., Cantarella, A., Franck, L., Meek, J., \& Fitzgerald, M. (2008). How well do clinical pain assessment tools reflect pain in infants? Public Library of Science Medicine, 5(6): e129. doi:10.1371/journal.pmed.0050129.

Soh, C. R., Ng, S. B. A., \& Lim, S. L. (2003). Dorsal penile nerve block. Pediatric Anesthesia, 13(4), 329-333.

Sorensen, E., Carskadon, M. A., \& Ursin, R. (2007). Sleep across the life cycle. In Butkov, N. and LeeChiong, T. (eds.), Fundamentals of Sleep Technology. Philadelphia, PA: Lippincott, Williams, \& Wilkins.

Stang, H. J., Gunnar, M. R., Snellman, L., Condon, L. M., \& Kestenbaum, R. (1988). Local anesthesia for neonatal circumcision: Effects on distress and cortisol response. Journal of the American Medical Association, 259(10), 1507-1511.

Stang, H. J., Snellman, L. W., Condon, L. M., Conroy, M. M., Liebo, R., Brodersen, L., \& Gunnar, M. R. (1997). Beyond dorsal penile nerve block: A more humane circumcision. Pediatrics, 100(2), doi: 10.1542/peds.100.2.e3.Taddio, A. (2001). Pain management for neonatal circumcision. Pediatric Drugs, 3(2), 101-111.

Taddio, A., Katz, J., Ilersich, A. L., \& Koren, G. (1997). Effect of neonatal circumcision on pain response during subsequent routine vaccination. The Lancet, 349, 599-603. 
Talbert, L. M., Kraybill, E. N., \& Potter, H. D. (1976). Adrenal cortical response to circumcision in the neonate. Obstetrics and Gynecology, 48(2), 208-210.

Thoman, E. B. (1990). Sleeping and waking states in infants: a functional perspective. Neuroscience and Biobehavioral Reviews. 14(1), 93-107.

Thoman, E. B., \& Glazier, R. C. (1987). Computer scoring of motility patterns for states of sleep and wakefulness: Human infants. Sleep, 10(2), 122-129.

Thoman, E. B., Korner, A. F., \& Kraemer, H. C. (1976). Individual consistency in behavioral states in neonates. Developmental Psychobiology, 9(3), 271-283.

Thoman, E. B., \& Tynan, W. (1979). Sleep states and wakefulness in human infants: Profiles from motility monitoring. Physiology \& Behavior, 23(3), 519-525.

Tarullo, A. R., Balsam, P. D., \& Fifer, W. P. (2011). Sleep and infant learning. Infant and Child Development, 20(1), 35-46. doi:10.1002/icd.685

Van Dongen, H. P. A., Maislin, G., Mullington, J. M., \& Dinges, D. F. (2003). The cumulative cost of additional wakefulness: Dose-response effects on neurobehavioral functions and sleep physiology from chronic sleep restriction and total sleep deprivation. Sleep, 26(2), 117-126.

Vetrivelan, R, Fuller, P. M., Tong, Q., \& Lu, J. (2009). Medullary circuitry regulating rapid eye movement sleep and motor atonia. Journal of Neuroscience, 29(29), 9361-9369.

Weisman, O., Magori-Cohen, R., Louzoun, Y., Eidelman, A., \& Feldman, R. (2011). Sleep-wake transitions in premature neonates predict early development. Pediatrics, 128(4), 706-714.

World Health Organization (2010). Manual for early infant male circumcision under local anesthesia. Johns Hopkins University affiliate. WHO document production services, Geneva, Switzerland. 


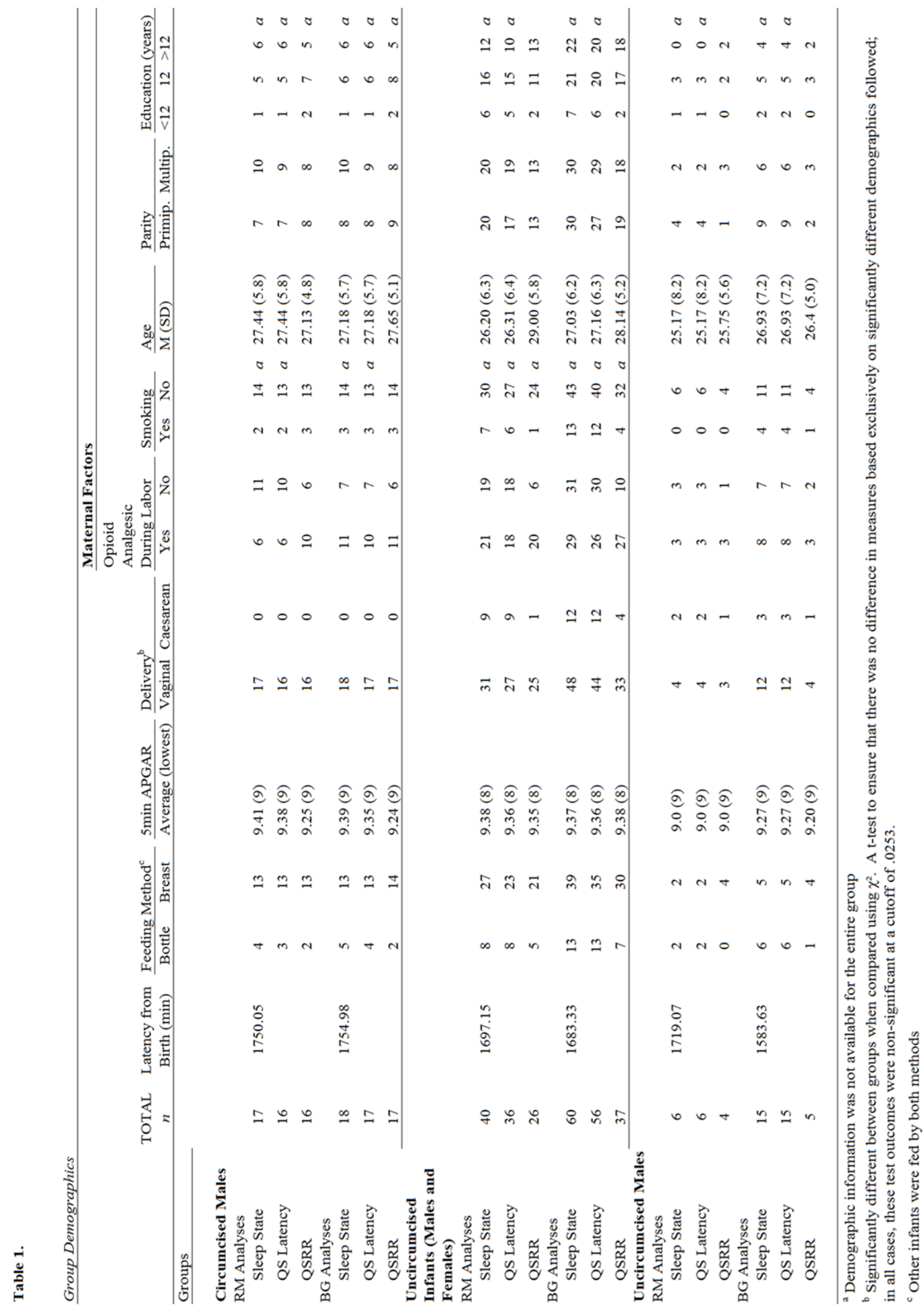




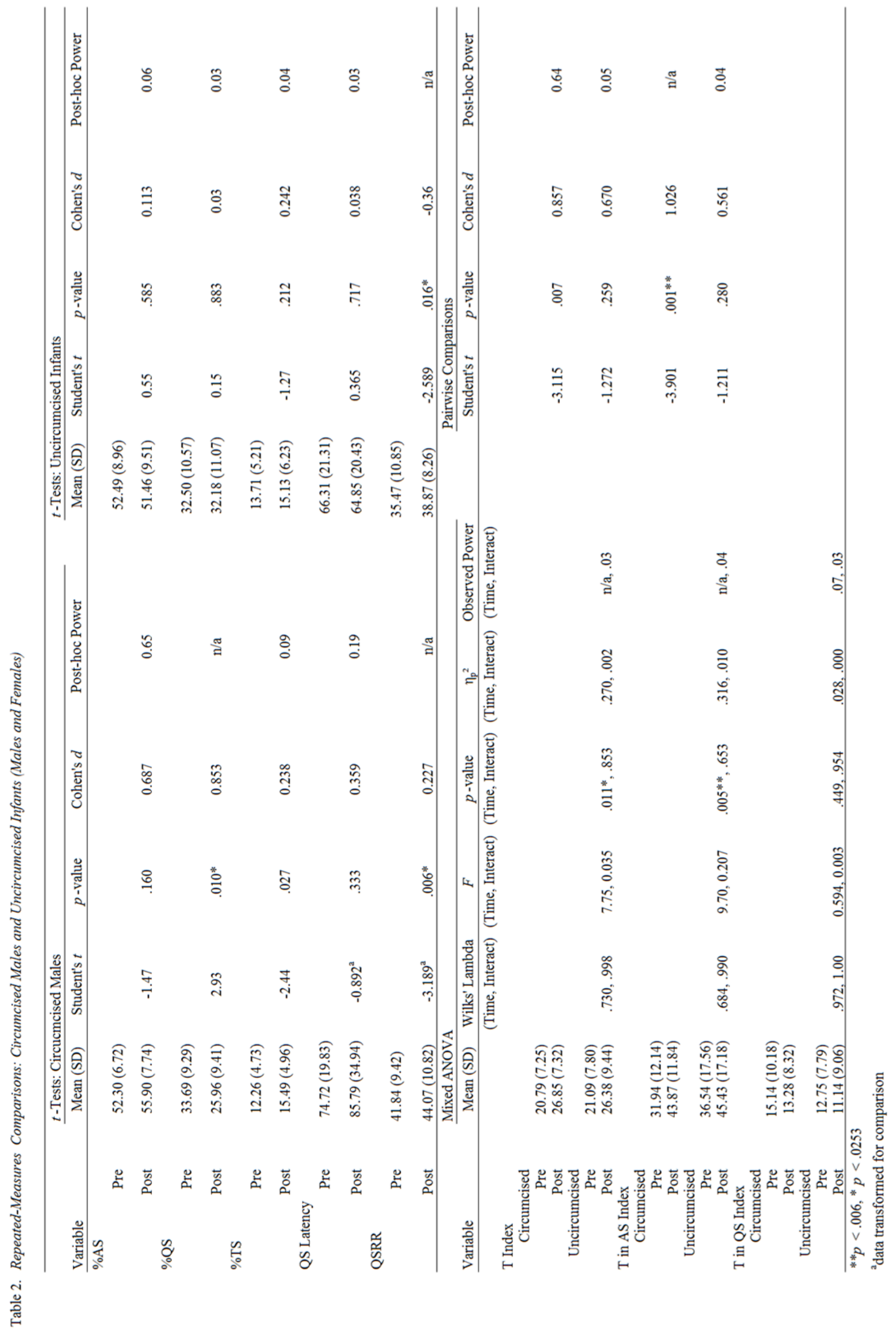




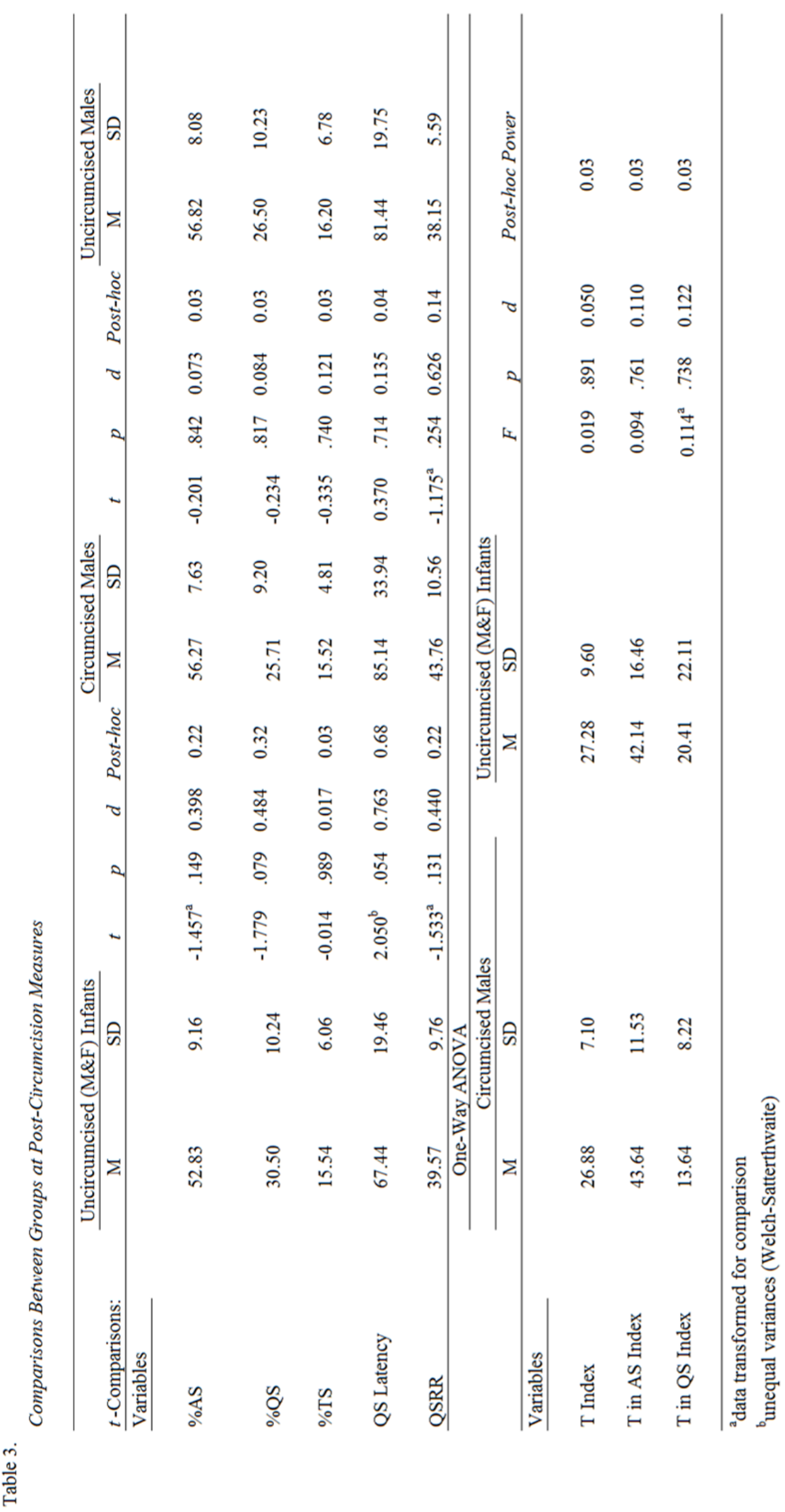




\section{Figure Captions}

Figure 1. Legend of abbreviations.

Figure 2. Sample sizes and exclusion.

Figure 3. Average proportion of QS in a sleep cycle for three groups of infants. Bars represent standard error of the mean. Repeated-measures sample sizes are reported in the legend. T-test outcomes of independent mean comparisons are depicted to the right of the legend; circumcised $n=18$, uncircumcised male-and-female $n=60 .{ }^{*} p<.0253$.

Figure 4. Average proportion of TS in a sleep cycle for three groups of infants. Bars represent standard error of the mean. Repeated-measures sample sizes are reported in the legend. T-test outcomes of independent mean comparisons are depicted to the right of the legend; circumcised $n=18$, uncircumcised male-and-female $n=60 .{ }^{\wedge} p=.027$.

Figure 5. Average latency to QS in a sleep cycle for three groups of infants. Bars represent standard error of the mean. Repeated-measures sample sizes are reported in the legend. T-test outcomes of independent mean comparisons are depicted to the right of the legend; circumcised $n=17$, uncircumcised male-andfemale $n=56 .{ }^{*} p<.0253$.

Figure 6. Shows the three variables evaluated using Mixed 2x2 ANOVAs in relation to one another. Bars represent standard error of the mean. The omnibus effect of time (pre- to-post) reached statistical significance for overall $(p=.010)$ and AS $(p=.005)$ measures while the interaction between time and circumcision group was not significant for any measure $\left(n_{p}{ }^{2}=.002, .01\right.$, and .00 , respective to the figure). Pairwise comparisons follow in Figures 8 and 9. No female infants were included in transition analyses. Figure 7. Post-hoc pairwise comparisons following the Mixed 2x2 ANOVA for the average index of behavioral transitions in a sleep cycle. Bars represent standard error of the mean. Circumcised $n=17$; Uncircumcised $n=6$. No female infants were included in transition analyses. A One-way ANOVA evaluating differences in transition index post-circumcision was not statistically significant (Circumcised $n=18$, Uncircumcised $n=15) .{ }^{\wedge} p=.007$, marginal statistical significance ( $p<.006$ cutoff). 
Figure 8. Post-hoc pairwise comparisons following the Mixed 2x2 ANOVA for the average index of behavioral transitions during AS. Bars represent standard error of the mean. Circumcised $n=17$; uncircumcised $n=6$. No female infants were included in transition analyses. A One-way ANOVA evaluating differences in transition index post-circumcision was not statistically significant (Circumcised $n=18$, Uncircumcised $n=15) . * p=.001$.

Figure 9. Average respiratory rate during QS for three groups of infants. Bars represent standard error of the mean. Repeated-measures sample sizes are reported in the legend. T-test outcomes of independent mean comparisons are depicted to the right of the legend; circumcised $n=17$, uncircumcised male-andfemale $n=37 . * p<.0253$.

Figure 10. Combined changes in sleep cycle organization among circumcised male infants. 
INFANT SLEEP ORGANIZATION

Legend: AS (Active Sleep), REM (Rapid Eye Movement), QS (Quiet Sleep), NREM (Non-Rapid Eye Movement), IS (Indeterminate Sleep), EEG (Electroencephalographic), EMLA (Eutectic Mixture of Local Anesthetics), SNS (Sympathetic Nervous System), HPA (Hypothalamic-Pituitary-Adrenal), TST (Total Sleep Time), SOL (Sleep-Onset Latency), NPT (Nocturnal Penile Tumescence), N1 and N2 (NREM Stage 1 and NREM Stage 2), RR (Respiratory Rate), SWS (Slow-Wave Sleep), Br/Min (Breaths per Minute), APGAR (Appearance, Pulse, Grimace, Activity, and Respiration), MMS (Motility Monitoring System) 


\section{Sample Size Derivation}

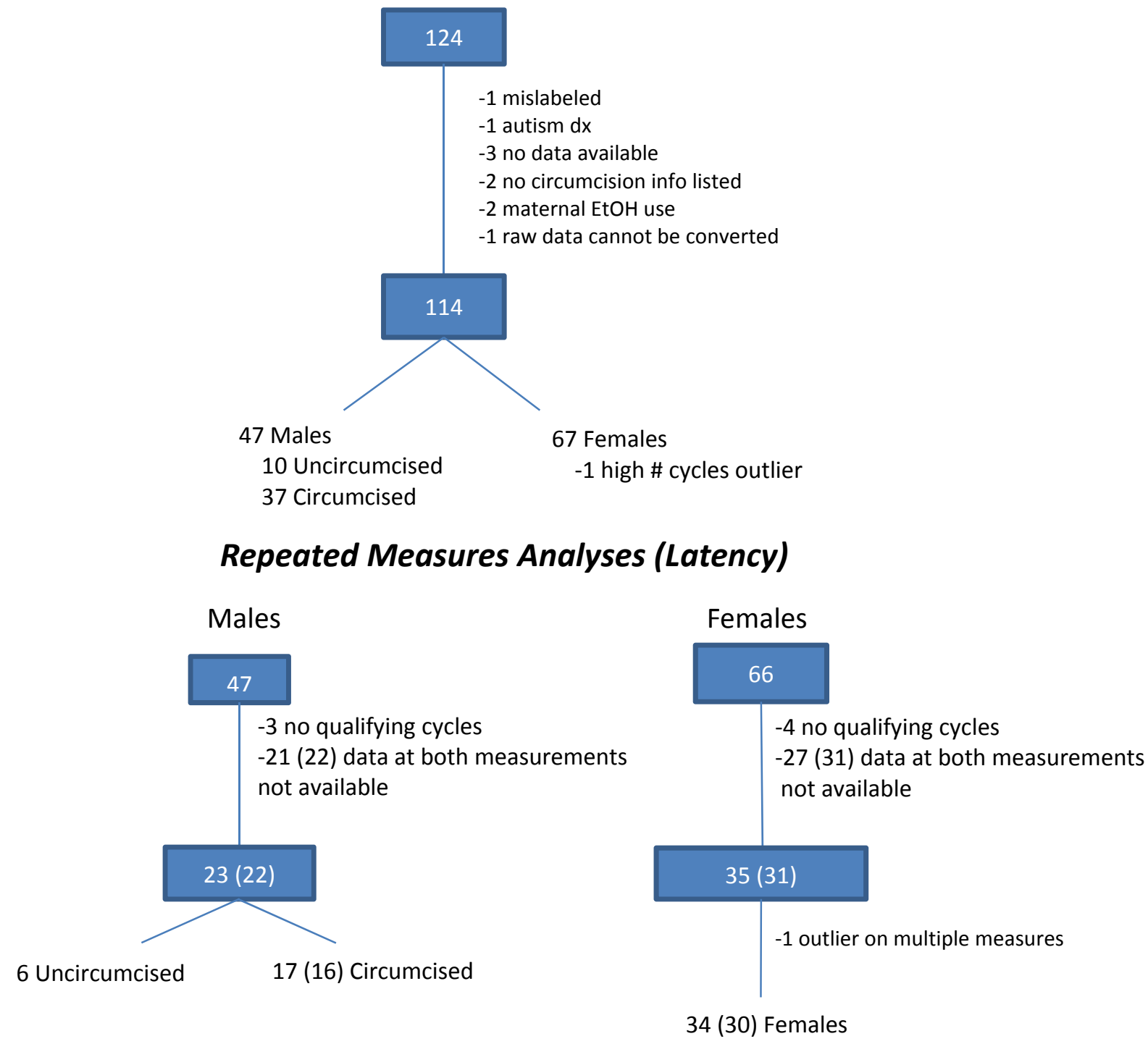

Between Groups Analyses (Latency)
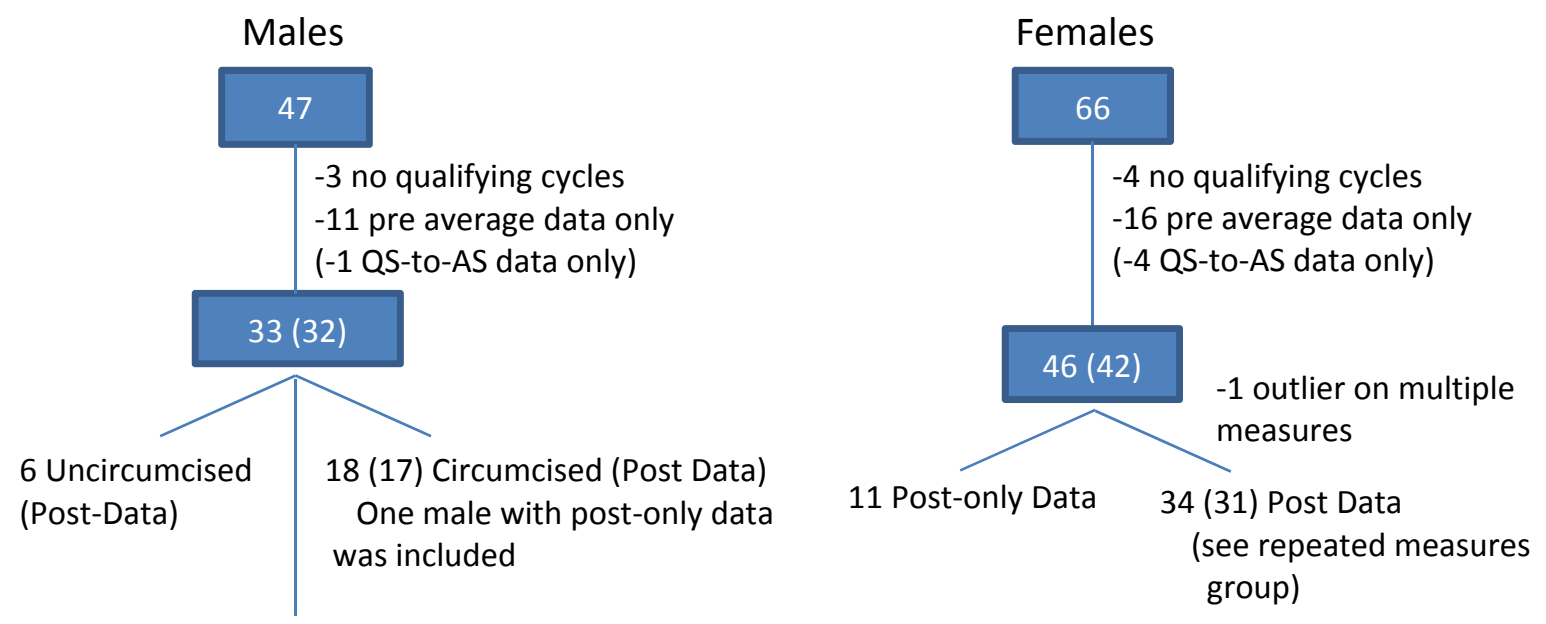

9 Pre-circumcision Post-average Data 


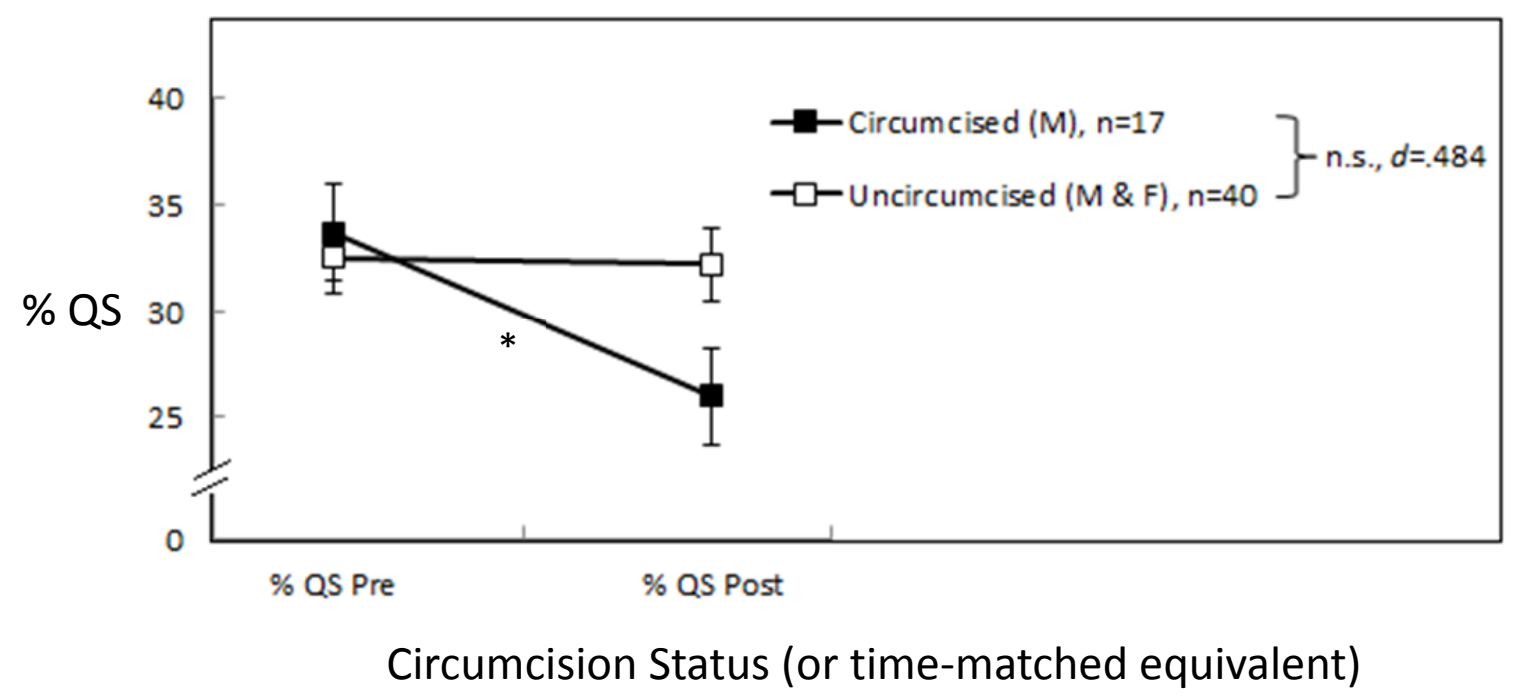




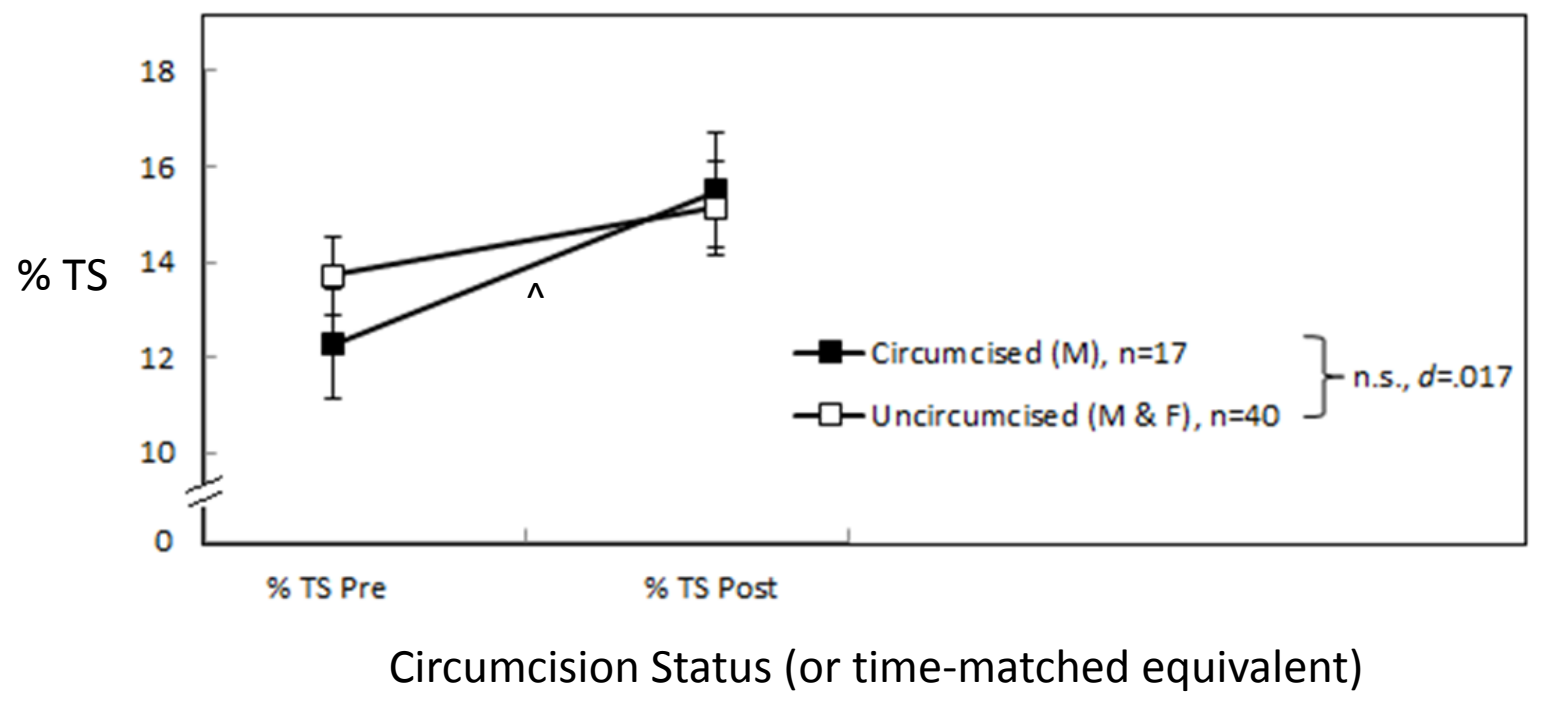




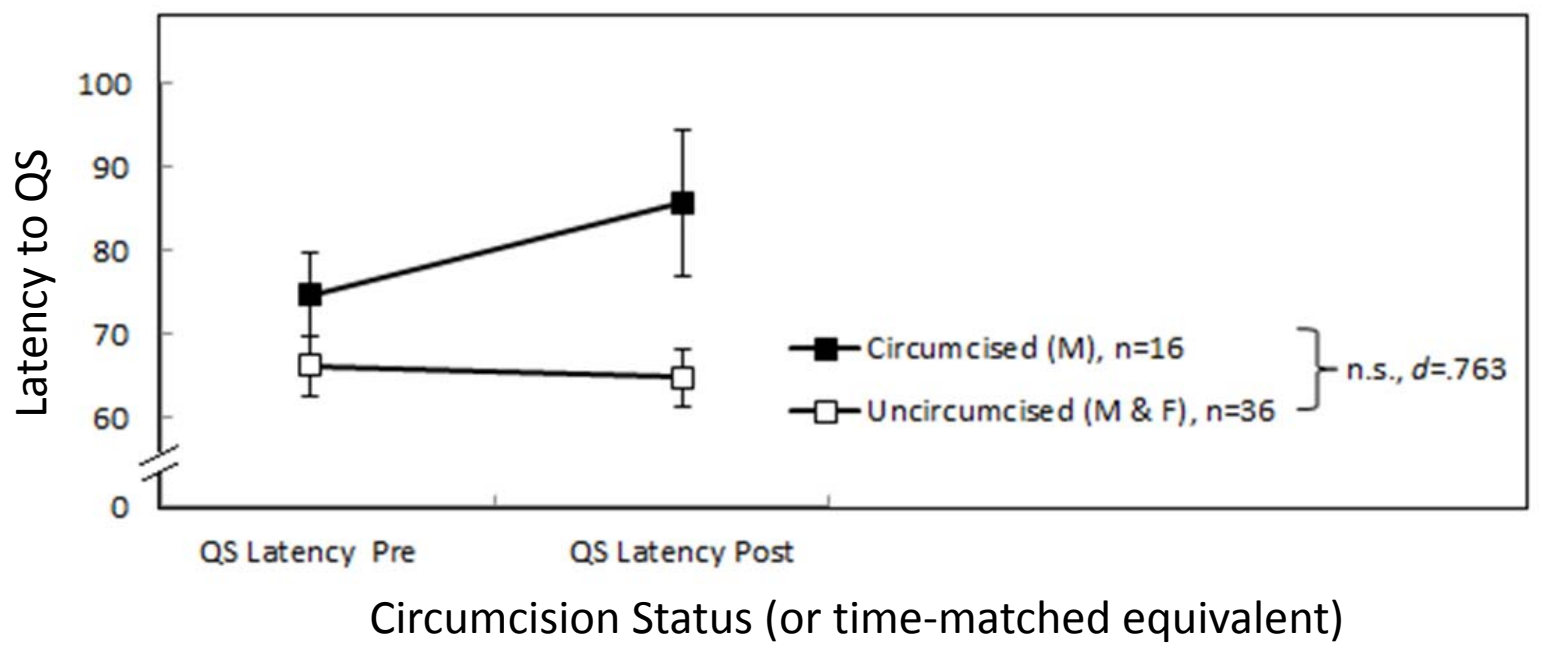




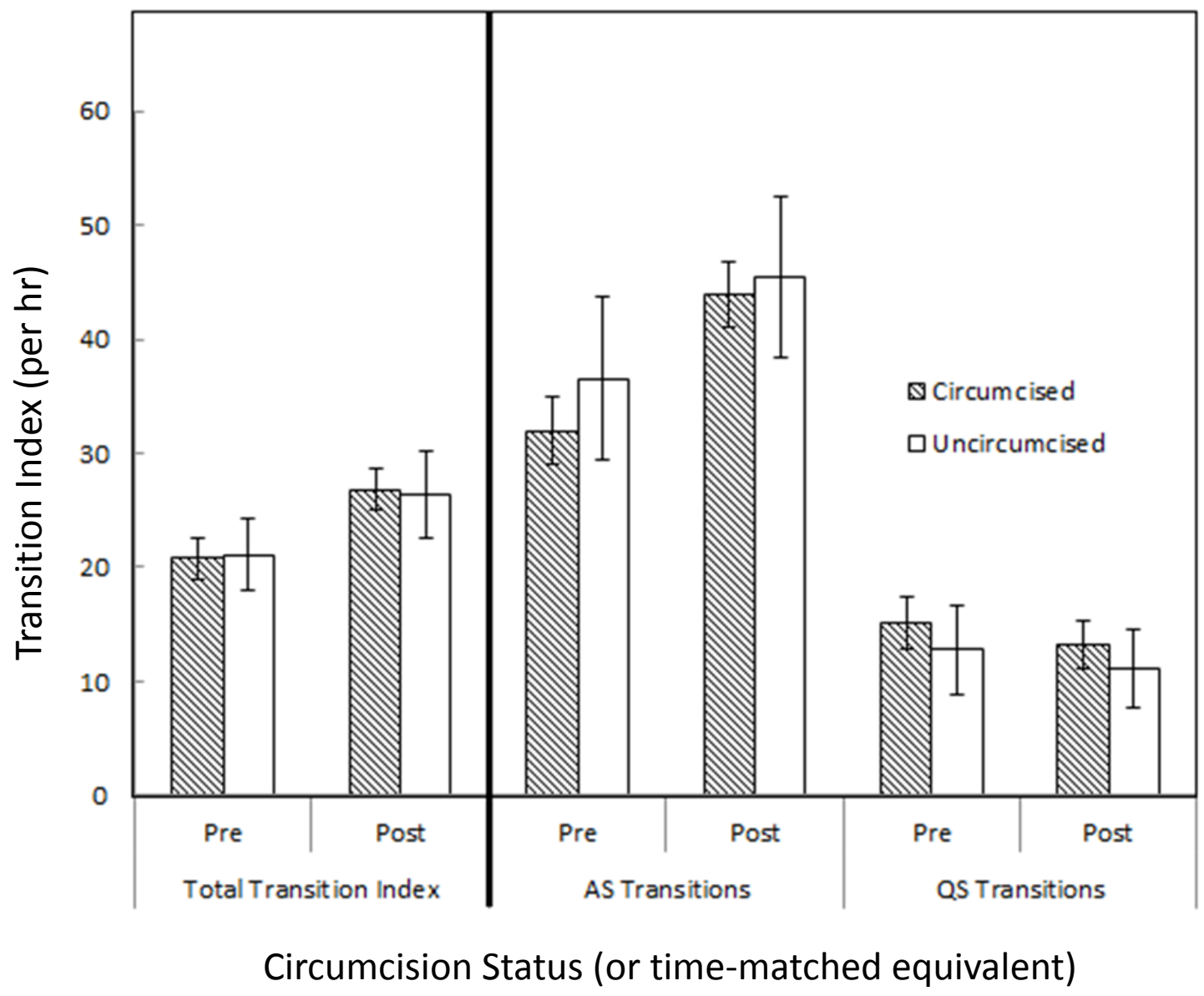




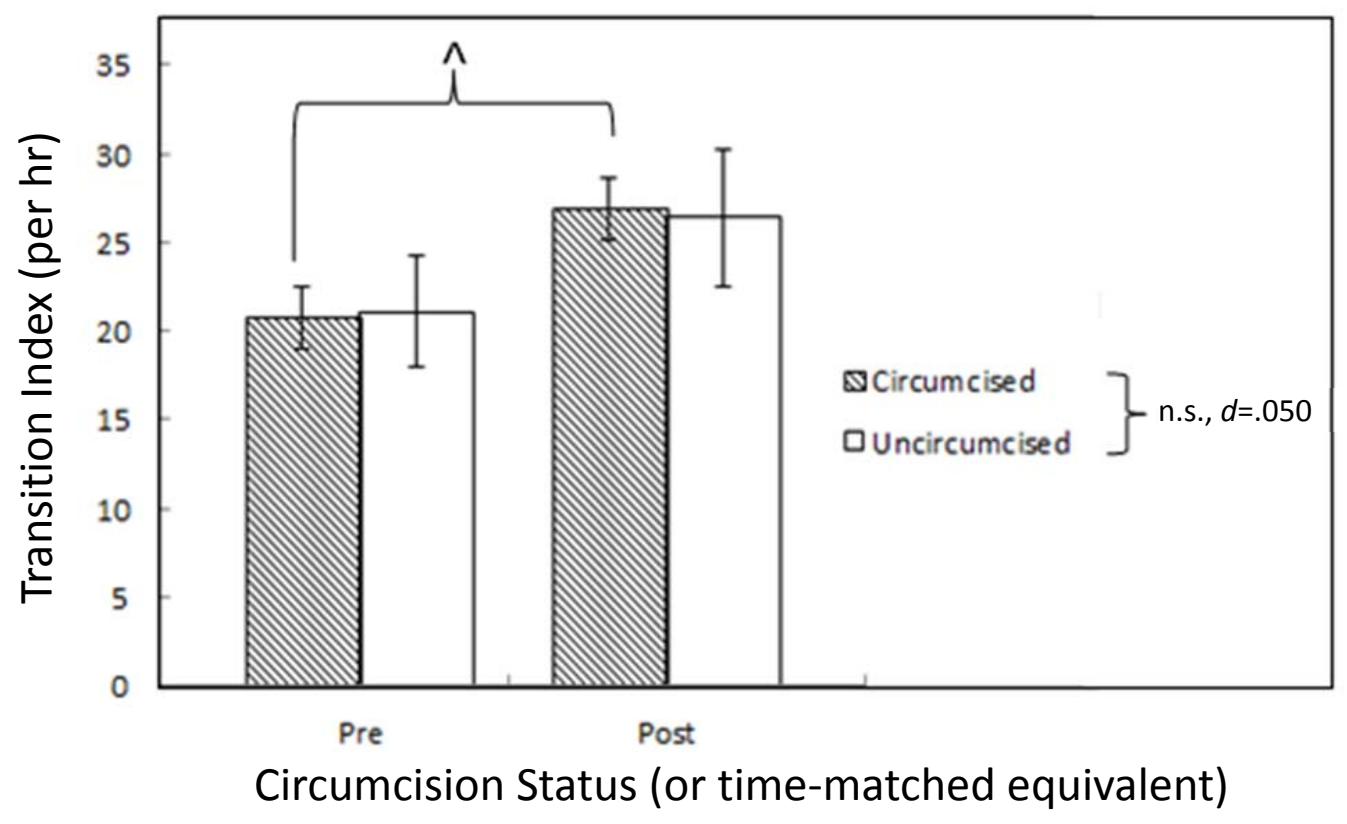




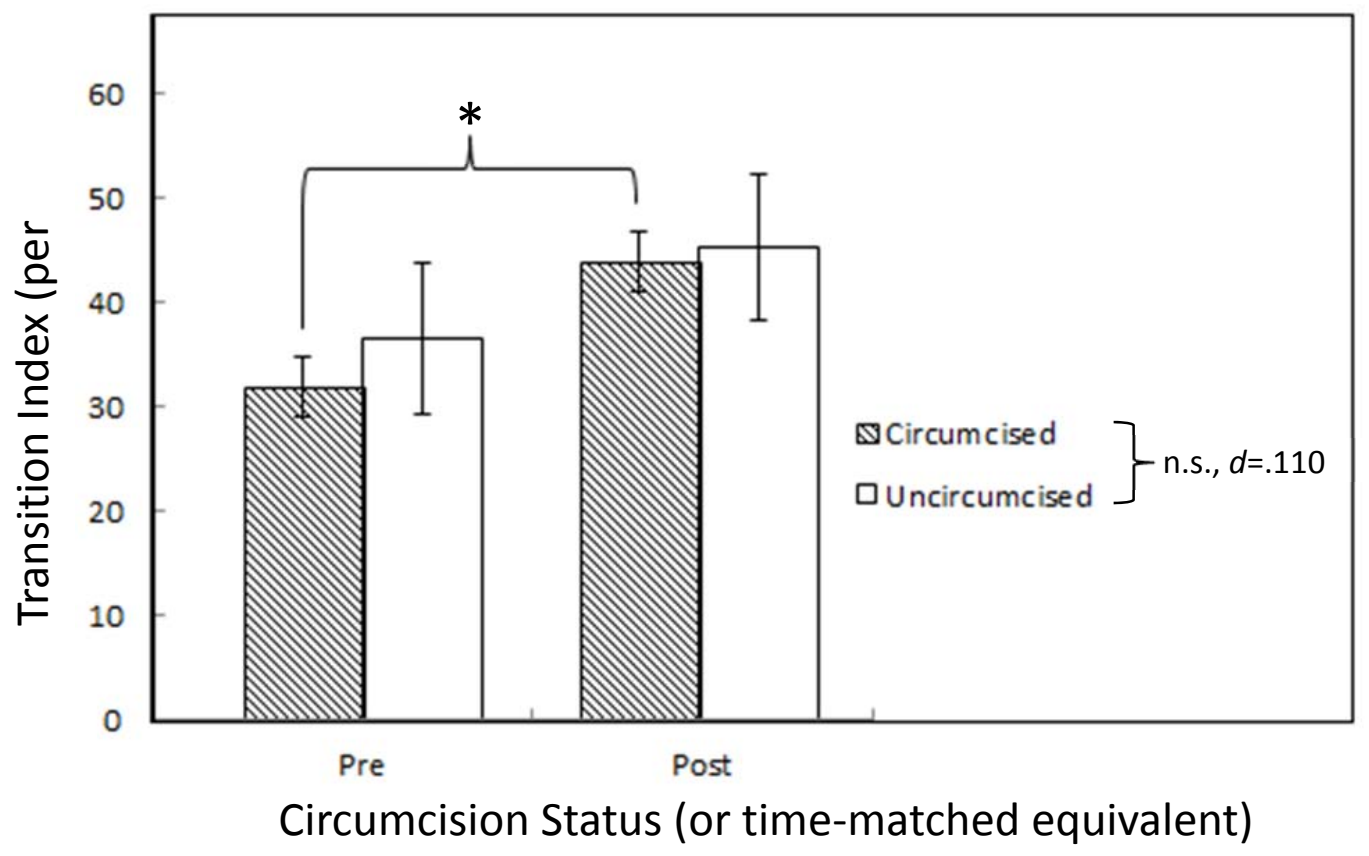




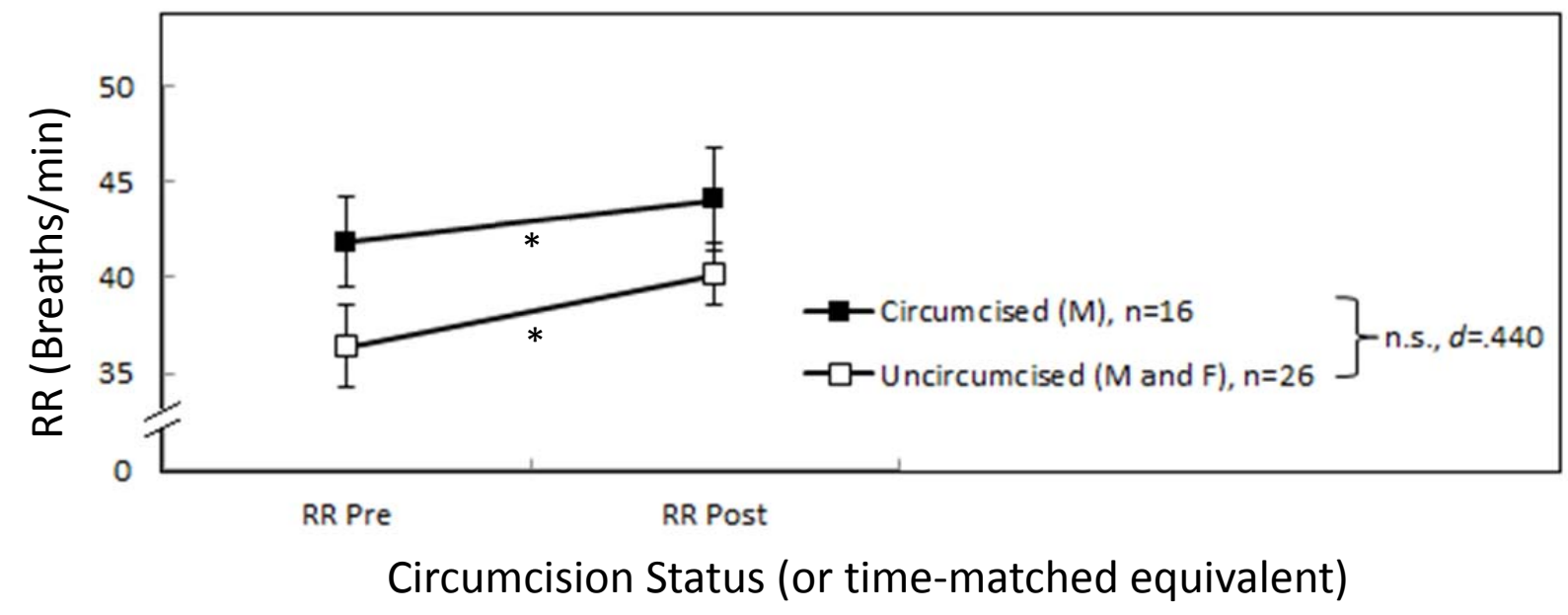


Combined sleep state organization changes by circumcised males.

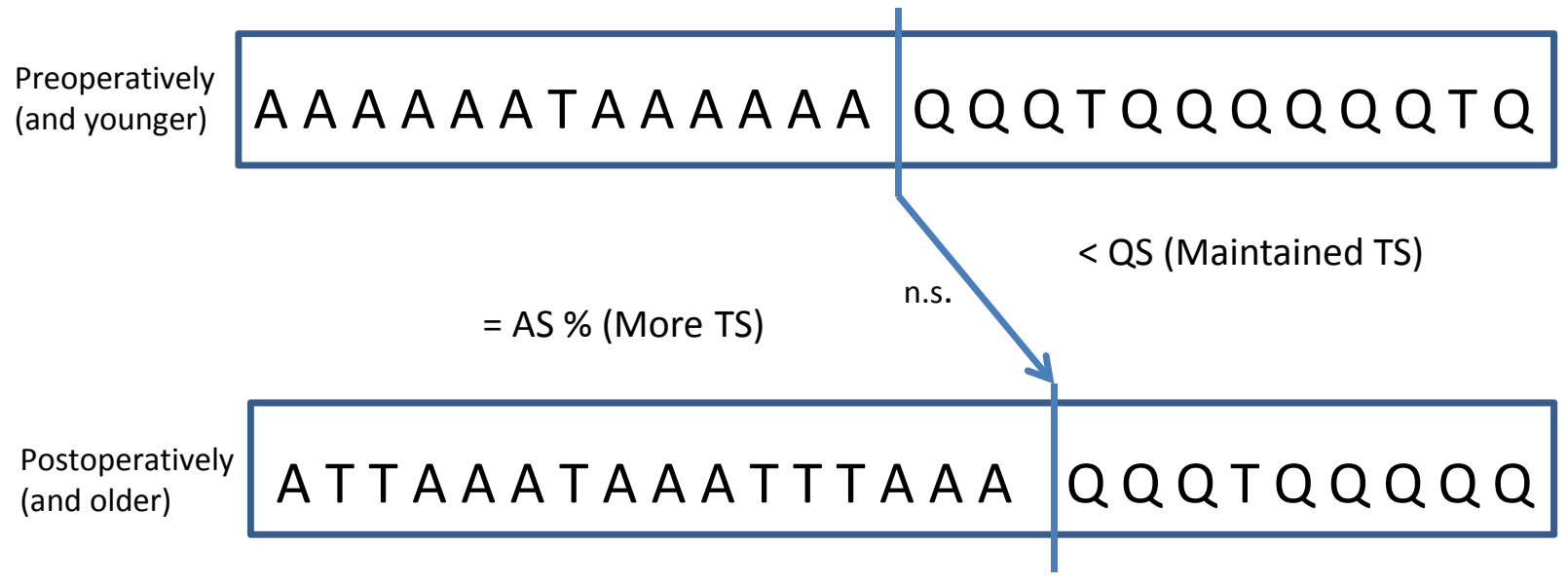

